TRANSACTIONS OF THE

AMERICAN MATHEMATICAL SOCIETY

Volume 348, Number 8, August 1996

\title{
PRESENTATION AND CENTRAL EXTENSIONS OF MAPPING CLASS GROUPS
}

\author{
SYLVAIN GERVAIS
}

\begin{abstract}
We give a presentation of the mapping class group $\mathcal{M}$ of a (possibly bounded) surface, considering either all twists or just non-separating twists as generators. We also study certain central extensions of $\mathcal{M}$. One of them plays a key role in studying TQFT functors, namely the mapping class group of a $p_{1}$-structure surface. We give a presentation of this extension.
\end{abstract}

\section{INTRODUCTION}

All surfaces that we shall consider are connected and oriented. We shall denote by $\Sigma_{g, r}$ a surface with genus $g$ and $r$ boundary components, or simply $\Sigma$ when there is no ambiguity. We shall denote its mapping class group by $\mathcal{M}_{g, r}$ (or $\mathcal{M}_{\Sigma}$ ). Recall that $\mathcal{M}_{\Sigma}$ is the group of diffeomorphisms of $\Sigma$ which leave fixed its boundary, modulo those which are isotopic to the identity. The aim of this article is to find a presentation of $\mathcal{M}_{g, r}$ with very simple geometrical relations and to describe more precisely this presentation as central extensions.

These groups are generated by Dehn twists $([\mathrm{D}])$. Lickorish ([L1, L2]) proved that $3 g-1$ of these twists generate $\mathcal{M}_{g, 0}$. Humphries ([Hu]) reduced this number to $2 g+1$ and showed that it was minimal.

Using the presentation of $\mathcal{M}_{g, 0}$ found by Hatcher and Thurston in [H-T], Wajnryb gave in [W] an explicit presentation of $\mathcal{M}_{g, 1}$ and $\mathcal{M}_{g, 0}$ with Humphries generators. We shall also use their result to obtain a presentation of $\mathcal{M}_{g, r}$ with the set of all Dehn twists for generators.

Four types of relations will appear in this presentation: "braids", "lanterns", "stars" and "chains".

The braids. It is well known that if $\alpha$ is a simple closed curve (s.c.c.) in $\Sigma$ and $h$ is a diffeomorphism of $\Sigma$, then $\tau_{h(\alpha)}=h \tau_{\alpha} h^{-1}$ (where $\tau_{\alpha}$ represents the twist along $\alpha$ ). More specifically, if $h$ is a twist $\tau_{\beta}$, one has the relation (called a braid relation)

$$
\tau_{\gamma}=\tau_{\beta} \tau_{\alpha} \tau_{\beta}^{-1}
$$

where $\gamma=\tau_{\beta}(\alpha)$. We will denote this relation by $T_{n}$ when $|\alpha \cap \beta|=n$ and by $T_{2_{0}}$ when $|\alpha \cap \beta|=2$ and the algebraic intersection is zero (denoted $|\alpha \cap \beta|=2_{0}$ ).

Remark. If $G$ is a multiplicative group, $\bar{x}$ will denote $x^{-1}$ and $y(x)$ will denote the conjugate of $x$ by $y$ (i.e. $y x \bar{y}$ ).

Received by the editors May 25, 1994 and, in revised form, April 5, 1995.

1991 Mathematics Subject Classification. Primary 57N05, 20F05; Secondary 20F34, 20F38, $57 \mathrm{M} 05$.

Key words and phrases. Mapping class groups, $p_{1}$-structure, central extensions, presentation. 


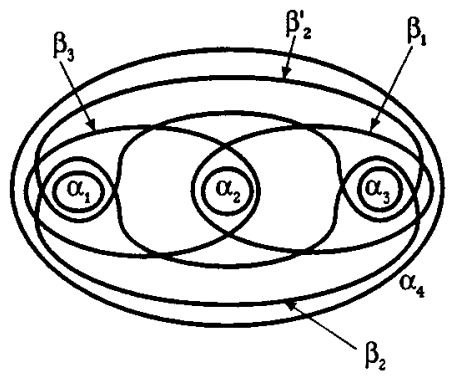

FiguRE 1

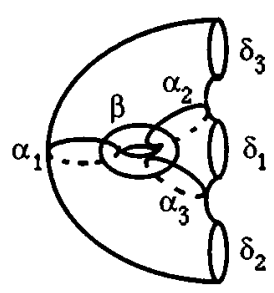

Figure 2

The lanterns. These relations were discovered by Johnson ([J]). Let us consider a subsurface of $\Sigma_{g, r}$ which is homeomorphic to a sphere with four holes. Let $\alpha_{1}$, $\alpha_{2}, \alpha_{3}$ and $\alpha_{4}$ be the boundary components and $\beta_{1}, \beta_{2}$ and $\beta_{3}$ curves as shown in Figure 1. The relation (called a lantern relation) is:

$$
\tau_{\alpha_{1}} \tau_{\alpha_{2}} \tau_{\alpha_{3}} \tau_{\alpha_{4}}=\tau_{\beta_{3}} \tau_{\beta_{2}} \tau_{\beta_{1}} .
$$

Remark. By a homology calculation, one can check that if two curves $\alpha$ and $\beta$ in a surface $\Sigma$ are such that $|\alpha \cap \beta|=2_{0}$, then a neighborhood of $\alpha \cup \beta$ in $\Sigma$ is homeomorphic to a sphere with four holes. So, a lantern $(L)$ is completely determined by the curves $\beta_{1}$ and $\beta_{3}$. The curves $\alpha_{i}$ are the four boundary components of a neighborhood of $\beta_{1} \cup \beta_{3}$. There are two choices for $\beta_{2}$, but only one gives the equality $(L)$ (the other choice, the curve $\beta_{2}^{\prime}$ shown in Figure 1, gives the equality $\tau_{\alpha_{1}} \tau_{\alpha_{2}} \tau_{\alpha_{3}} \tau_{\alpha_{4}}=\tau_{\beta_{1}} \tau_{\beta_{2}^{\prime}} \tau_{\beta_{3}}$ ).

The stars. We consider a subsurface of $\Sigma_{g, r}$ which is homeomorphic to a surface of genus one with three boundary components. The relation is

$$
\left(\tau_{\alpha_{1}} \tau_{\alpha_{2}} \tau_{\alpha_{3}} \tau_{\beta}\right)^{3}=\tau_{\delta_{1}} \tau_{\delta_{2}} \tau_{\delta_{3}},
$$

where the curves are described in Figure 2.

Remark. The star $(E)$ is uniquely determined by four curves $\alpha_{1}, \alpha_{2}, \alpha_{3}$ and $\beta$ such that $\left|\alpha_{i} \cap \alpha_{j}\right|=0$ if $i \neq j$ and $\left|\beta \cap \alpha_{i}\right|=1$ for all $i \in\{1,2,3\}$ (a neighborhood of these curves in $\Sigma$ is homeomorphic to $\Sigma_{1,3}$ ).

The chains. These are just a particular case of the stars: we suppose that the curve $\delta_{1}$ bounds a disc in $\Sigma$. Then, we have $\tau_{\delta_{1}}=1, \tau_{\alpha_{2}}=\tau_{\alpha_{3}}$, and the relation $(E)$ becomes:

$$
\left(\tau_{\alpha_{1}} \tau_{\beta} \tau_{\alpha_{2}}\right)^{4}=\tau_{\delta_{2}} \tau_{\delta_{3}}
$$


Remark. Using relations $T_{0}$ and $T_{1}$, it is easy to show that $\left(\tau_{\alpha_{1}} \tau_{\alpha_{2}} \tau_{\alpha_{2}} \tau_{\beta}\right)^{3}=$ $\left(\tau_{\alpha_{1}} \tau_{\beta} \tau_{\alpha_{2}}\right)^{4}$. This kind of calculation will be often done in this article, and the reader can refer to them. Note that a chain is determined by the curves $\alpha_{1}, \beta$ and $\alpha_{2}$, with $\left|\alpha_{i} \cap \beta\right|=1$ and $\alpha_{1} \cap \alpha_{2}=\emptyset$.

Theorem A. For any oriented surface $\Sigma$ of genus $g \geq 0$, with $r$ boundary components $(r \geq 0), \mathcal{M}_{\Sigma}$ has the following presentation:

generators: $\left\{\tau_{\alpha} / \alpha\right.$ s.c.c. in $\left.\Sigma\right\}$ (if $\alpha$ bounds a disc in $\Sigma$, then $\tau_{\alpha}=1$ ).

relations: (I) All the braids $T_{0}, T_{1}$ and $T_{2_{0}}$.

(II) All the chains.

(III) All the lanterns.

If $g \geq 1$, one can replace (II) and (III) by (IV) :

(IV) All the stars.

Remark. If $g=0$, only the relations $T_{0}, T_{2_{0}}$ and (III) remain.

Harer proved in $[\mathrm{H}]$ that when $g \geq 3, \mathcal{M}_{g, r}$ is generated by the twists along the non-separating curves for any $r$. We shall see that this result is still true when $g=2$, but not when $g=1$. We shall also give a presentation of $\mathcal{M}_{g, r}(g \geq 2)$ with these generators.

Notation. If the curves involved in a lantern $L$ (resp. a star $E$ or a chain $C$ ) are all non-separating curves, we will denote $L$ by $L_{0}$ (resp. $E$ by $E_{0}$ and $C$ by $C_{0}$ ).

Theorem B. For any orientable surface $\Sigma$ of genus $g \geq 2$, with $r$ boundary components $(r \geq 0), \mathcal{M}_{\Sigma}$ has the following presentation:

generators: $\left\{\tau_{\alpha} / \alpha\right.$ non-separating s.c.c. in $\left.\Sigma\right\}$.

relations: (I)' All the braids $T_{0}$ and $T_{1}$ (with non-separating curves).

(II)' One chain $C_{0}$.

(III)' One lantern $L_{0}$.

When $g \geq 3$, one can replace (II)' or (III)' by a star $E_{0}$.

Remark. In the case of genus two, there are only the relations (I)' and (II)' (the lanterns $L_{0}$ and the stars $E_{0}$ exist only if $g \geq 3$ ).

Let $S_{g, r}$ be the group generated by $\left\{a_{\alpha} / \alpha\right.$ non-separating s.c.c. in $\left.\Sigma\right\}$ and defined by the relations $T_{0}$ and $T_{1}$ between these curves. Theorem B shows that $\mathcal{M}_{g, r}$ is a quotient of $S_{g, r}$ when $g \geq 2$. We shall prove the following more precise result.

Theorem C. For any orientable surface $\Sigma$, there exists a central extension

$$
0 \longrightarrow \mathbb{Z} \longrightarrow \mathcal{T}_{\Sigma} \longrightarrow \mathcal{M}_{\Sigma} \longrightarrow 1
$$

satisfying the following properties:

1) $\Sigma \mapsto \mathcal{M}_{\Sigma}, \Sigma \mapsto S_{\Sigma}$ and $\Sigma \mapsto \mathcal{T}_{\Sigma}$ are three functors from the category of surfaces (where morphisms are embeddings) to the category of groups, and the extension $(*)$ is natural. When $g \geq 3$ ( $g$ denotes the genus of $\Sigma$ ), this extension is the universal central extension of $\mathcal{M}_{\Sigma}$.

Furthermore, there exist two natural morphisms $\varphi: S_{\Sigma} \rightarrow \mathcal{T}_{\Sigma}$ and $\psi: S_{\Sigma} \rightarrow \mathbb{Z}$ such that:

2) if $g \geq 3, \varphi$ and $\psi$ induce an isomorphism from $S_{\Sigma}$ to $\mathcal{T}_{\Sigma} \times \mathbb{Z}$.

3) if $g=2, \varphi$ is an isomorphism from $S_{\Sigma}$ to $\mathcal{T}_{\Sigma}$. 


\section{Preliminary Results}

In this section, $\Sigma$ is a surface of genus $g \geq 0$ with $r$ boundary components $(r \geq 0)$.

Let $R_{\Sigma}$ (resp. $S_{\Sigma}$ ) be the group generated by $\left\{a_{\alpha} / \alpha\right.$ a s.c.c. in $\left.\Sigma\right\}$ (resp. $\left\{a_{\alpha} / \alpha\right.$ a non-separating s.c.c. in $\left.\left.\Sigma\right\}\right)$ and defined by the relations $T_{0}, T_{1}$ and $T_{2_{0}}$ (resp. the relations $T_{0}$ and $T_{1}$ between non-separating curves). The goal of this section will be to prove that all the braid relations are satisfied in $R$ and $S$ and to find an equality between the lanterns, the stars and the chains.

We start by proving two lemmas that will be used often.

Lemma 1.1. (i) Let $\alpha$ and $\beta$ be two disjoint s.c.c. (resp. non-separating s.c.c.) in $\Sigma$. Then, $a_{\alpha}$ commutes with $a_{\beta}$ in $R$ (resp. $S$ ).

(ii) If $\alpha$ and $\beta$ are two s.c.c. in $\Sigma$ such that $|\alpha \cap \beta|=1$, then $a_{\alpha} a_{\beta} a_{\alpha}=a_{\beta} a_{\alpha} a_{\beta}$ in $R$ and $S$.

Notation. In the following, we will refer to these relations respectively by (0) and (1).

Proof. ( $i)$ is exactly the relation $T_{0}$ because $\tau_{\beta}(\alpha)=\alpha$. (ii) is a consequence of $T_{1}$ and the fact that $\tau_{\beta}(\alpha)=\tau_{\alpha}^{-1}(\beta)$.

Lemma 1.2. Let $\left(\alpha_{1}, \alpha_{2}, \alpha_{3}, \beta\right)$ be a star in $\Sigma$ and set

$$
X_{1}=a_{\alpha_{1}} a_{\alpha_{2}}, \quad X_{2}=a_{\beta} X_{1} a_{\beta}, \quad X_{3}=a_{\alpha_{3}} X_{2} a_{\alpha_{3}} .
$$

Then, one has in $R$ and $S$ :

$$
\begin{aligned}
& \text { (i) } X_{i} X_{j}=X_{j} X_{i}, \\
& \text { (ii) }\left(a_{\alpha_{1}} a_{\alpha_{2}} a_{\alpha_{3}} a_{\beta}\right)^{3}=X_{1} X_{2} X_{3} \text {, } \\
& \text { (iii) }\left(a_{\alpha_{1}} a_{\beta} a_{\alpha_{2}}\right)^{4}=X_{1}^{2} X_{2}^{2} \text {. }
\end{aligned}
$$

Proof. (i) Using (1), we obtain

$$
a_{\alpha_{1}} X_{2}=a_{\alpha_{1}} a_{\beta} a_{\alpha_{1}} a_{\alpha_{2}} a_{\beta}=a_{\beta} a_{\alpha_{1}} a_{\beta} a_{\alpha_{2}} a_{\beta}=a_{\beta} a_{\alpha_{1}} a_{\alpha_{2}} a_{\beta} a_{\alpha_{2}}=X_{2} a_{\alpha_{2}}
$$

and in the same way, $a_{\alpha_{2}} X_{2}=X_{2} a_{\alpha_{1}}$. From this, we conclude that $X_{1} X_{2}=X_{2} X_{1}$ and, since $a_{\alpha_{3}}$ commutes with $a_{\alpha_{1}}$ and $a_{\alpha_{2}}$ (by (0)), $X_{1} X_{3}=X_{3} X_{1}$.

One has by (0) and (1)

$$
\begin{aligned}
a_{\beta} X_{3} & =a_{\beta} a_{\alpha_{3}} a_{\beta} a_{\alpha_{1}} a_{\alpha_{2}} a_{\beta} a_{\alpha_{3}}=a_{\alpha_{3}} a_{\beta} a_{\alpha_{3}} a_{\alpha_{1}} a_{\alpha_{2}} a_{\beta} a_{\alpha_{3}} \\
& =a_{\alpha_{3}} a_{\beta} a_{\alpha_{1}} a_{\alpha_{2}} a_{\beta} a_{\alpha_{3}} a_{\beta}=X_{3} a_{\beta}
\end{aligned}
$$

so $X_{2}$ commutes with $X_{3}$.

$$
\begin{aligned}
& \text { (ii) } X_{1} X_{2} X_{3}=a_{\alpha_{1}} a_{\alpha_{2}} a_{\beta} a_{\alpha_{1}} a_{\alpha_{2}} a_{\beta} a_{\alpha_{3}} a_{\beta} a_{\alpha_{1}} a_{\alpha_{2}} a_{\beta} a_{\alpha_{3}} \\
& =a_{\alpha_{1}} a_{\alpha_{2}} a_{\beta} a_{\alpha_{1}} a_{\alpha_{2}} a_{\alpha_{3}} a_{\beta} a_{\alpha_{3}} a_{\alpha_{1}} a_{\alpha_{2}} a_{\beta} a_{\alpha_{3}} \text { by (1) } \\
& =a_{\alpha_{1}} a_{\alpha_{2}} a_{\beta} a_{\alpha_{3}} a_{\alpha_{1}} a_{\alpha_{2}} a_{\beta} a_{\alpha_{2}} a_{\alpha_{1}} a_{\alpha_{3}} a_{\beta} a_{\alpha_{3}} \text { by (0) } \\
& =a_{\alpha_{1}} a_{\alpha_{2}} a_{\beta} a_{\alpha_{3}} a_{\alpha_{1}} a_{\beta} a_{\alpha_{2}} a_{\beta} a_{\alpha_{1}} a_{\beta} a_{\alpha_{3}} a_{\beta} \text { by (1) } \\
& =a_{\alpha_{1}} a_{\alpha_{2}} a_{\beta} a_{\alpha_{3}} a_{\alpha_{1}} a_{\beta} a_{\alpha_{2}} a_{\alpha_{1}} a_{\beta} a_{\alpha_{1}} a_{\alpha_{3}} a_{\beta} \text { by (1) } \\
& =a_{\alpha_{1}} a_{\alpha_{2}} a_{\beta} a_{\alpha_{3}} a_{\beta} a_{\alpha_{2}} a_{\alpha_{1}} a_{\beta} a_{\alpha_{2}} a_{\alpha_{1}} a_{\alpha_{3}} a_{\beta} \text { by (2) } \\
& =\left(a_{\alpha_{1}} a_{\alpha_{2}} a_{\alpha_{3}} a_{\beta}\right)^{3} \text { by (0) and (1). } \\
& \text { (iii) } X_{1}^{2} X_{2}^{2}=a_{\alpha_{1}} a_{\alpha_{2}} a_{\beta} a_{\alpha_{1}} a_{\alpha_{2}} a_{\beta} a_{\alpha_{1}} a_{\alpha_{2}} a_{\beta} a_{\alpha_{1}} a_{\alpha_{2}} a_{\beta} \text { by }(i) \\
& =a_{\alpha_{1}} a_{\beta} a_{\alpha_{2}} a_{\beta} a_{\alpha_{1}} a_{\beta} a_{\alpha_{2}} a_{\beta} a_{\alpha_{1}} a_{\beta} a_{\alpha_{2}} a_{\beta} \text { by (0) and (1) } \\
& =a_{\alpha_{1}} a_{\beta} a_{\alpha_{2}} a_{\alpha_{1}} a_{\beta} a_{\alpha_{2}} a_{\alpha_{1}} a_{\beta} a_{\alpha_{1}} a_{\alpha_{2}} a_{\beta} a_{\alpha_{2}} \text { by (0) and (1) } \\
& =\left(a_{\alpha_{1}} a_{\beta} a_{\alpha_{2}}\right)^{4} \text { by }(0) \text {. }
\end{aligned}
$$


PRESENTATION AND CENTRAL EXTENSIONS OF MAPPING CLASS GROUPS 310

\subsection{The braids.}

Theorem 1.3. For any simple closed curves $\alpha$ and $\beta$ in $\Sigma$, one has in $R_{\Sigma} a_{\gamma}=$ $a_{\beta} a_{\alpha} \overline{a_{\beta}}$ where $\gamma=\tau_{\beta}(\alpha)$.

If $\alpha$ and $\beta$ do not separate, this relation is also true in $S_{\Sigma}$.

Corollary 1.4. Let $h$ be a diffeomorphism of $\Sigma$ and suppose that it is decomposed into a product of Dehn twists; denote by $m$ the corresponding word in $R_{\Sigma}$. Let $\alpha$ be a s.c.c. in $\Sigma$ and $\gamma=h(\alpha)$. Then, in $R_{\Sigma}$ one has the relation $a_{\gamma}=m a_{\alpha} \bar{m}$.

Furthermore, if the twists involved in the decomposition of $h$ are all twists along non-separating curves and if $\alpha$ does not separate, then the relation is also true in $S_{\Sigma}$.

Proof. Write $h=\tau_{\beta_{n}}^{\varepsilon_{n}} \ldots \tau_{\beta_{1}}^{\varepsilon_{1}}\left(\varepsilon_{i}= \pm 1\right.$ ); then, $m=a_{\beta_{n}}^{\varepsilon_{n}} \ldots a_{\beta_{1}}^{\varepsilon_{1}}$ in $R$ (or $S$ ).

Denote, for $k \in\{0, \ldots, n\}, \gamma_{k}=\tau_{\beta_{k}}^{\varepsilon_{k}} \ldots \tau_{\beta_{1}}^{\varepsilon_{1}}(\alpha)\left(\gamma_{0}=\alpha\right.$ and $\left.\gamma_{n}=\gamma\right)$. Then, Theorem 1.3 implies

$$
a_{\gamma_{k}}=a_{\beta_{k}}^{\varepsilon_{k}} a_{\gamma_{k-1}} a_{\beta_{k}}^{-\varepsilon_{k}}
$$

thus

$$
a_{\gamma}=a_{\gamma_{n}}=a_{\beta_{n}}^{\varepsilon_{n}} a_{\gamma_{n-1}} a_{\beta_{n}}^{-\varepsilon_{n}}=\ldots=m a_{\alpha} \bar{m} .
$$

Before we prove Theorem 1.3, let us show that the relations $T_{2_{0}}$ are true in $S$ :

Lemma 1.5. Let $\alpha$ and $\beta$ be two non-separating curves in $\Sigma$ such that $|\alpha \cap \beta|=2_{0}$. Then, if $\gamma=\tau_{\beta}(\alpha)$, one has in $S$

$$
a_{\gamma}=a_{\beta} a_{\alpha} \overline{a_{\beta}} .
$$

Proof. A neighborhood of $\alpha \cup \beta$ in $\Sigma$ is a lantern where $\alpha$ and $\beta$ are respectively identified with the curves $\beta_{1}$ and $\beta_{2}$ of the Figure 1. Since these curves do not separate, there exists a curve $\delta$ such that

$$
|\delta \cap \alpha|=|\delta \cap \beta|=\left|\delta \cap \alpha_{1}\right|=\left|\delta \cap \alpha_{2}\right|=1 .
$$

(We might have to replace $\left\{\alpha_{1}, \alpha_{2}\right\}$ by $\left\{\alpha_{3}, \alpha_{4}\right\}$.)

It can be seen that

$$
\tau_{\delta} \tau_{\alpha_{1}} \tau_{\alpha_{2}} \tau_{\delta}(\beta)=\alpha \quad \text { and } \quad \tau_{\delta}^{-1} \tau_{\alpha_{1}}^{-1} \tau_{\alpha_{2}}^{-1} \tau_{\delta}^{-1}(\beta)=\gamma .
$$

And since $|\delta \cap \beta|=\left|\alpha_{2} \cap \tau_{\delta}(\beta)\right|=\ldots=\left|\delta \cap \tau_{\alpha_{1}}^{-1} \tau_{\alpha_{2}}^{-1} \tau_{\delta}^{-1}(\beta)\right|=1$, the relation $T_{1}$ implies

$$
X a_{\beta} \bar{X}=a_{\alpha} \quad \text { and } \quad \bar{X} a_{\beta} X=a_{\gamma}, \quad \text { where } \quad X=a_{\delta} a_{\alpha_{1}} a_{\alpha_{2}} a_{\delta}
$$

and so $a_{\beta} a_{\alpha} \overline{a_{\beta}}=a_{\beta} X a_{\beta} \bar{X} \overline{a_{\beta}}$.

Since $\left(\alpha_{1}, \alpha_{2}, \beta, \delta\right)$ defines a star in $\Sigma$, Lemma 1.2 shows that $X$ commutes with $a_{\beta} X a_{\beta}$. Thus, we obtain:

$$
a_{\beta} a_{\alpha} \overline{a_{\beta}}=\bar{X} a_{\beta} X a_{\beta} \overline{a_{\beta}}=a_{\gamma} .
$$

Proof of Theorem 1.3. Suppose inductively that the braid relations $T_{k}$ are satisfied in $R$ and $S$ for $k \leq n-1$, let $\alpha$ and $\beta$ be two s.c.c. in $\Sigma$ such that $|\alpha \cap \beta|=n$, and set $\gamma=\tau_{\beta}(\alpha)$ (for $S$, we suppose also that $\alpha$ and $\beta$ do not separate). Let us fix an orientation on $\alpha$ and $\beta$.

First case. There exist two points $A$ and $B$ in $\alpha \cap \beta$ which are consecutive on $\alpha$ and such that the signs of the intersection of $\alpha$ and $\beta$ at these points are the same. 


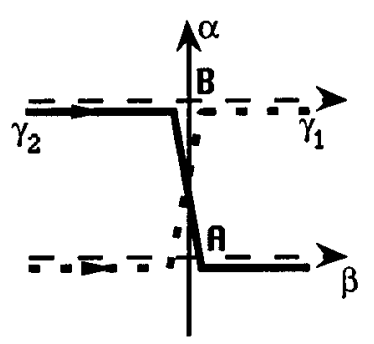

Figure 3

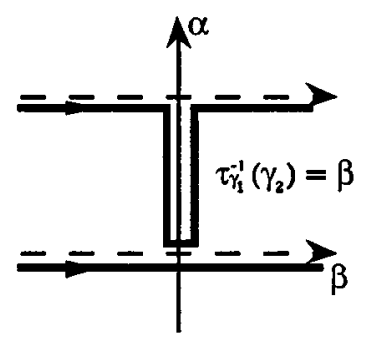

FiguRE 4

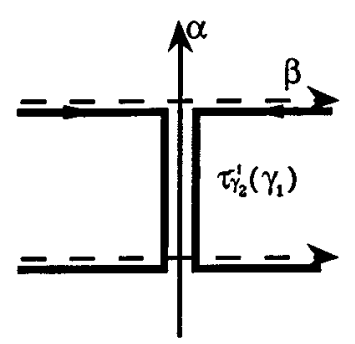

FiguRe 5

Let us consider the curves $\gamma_{1}$ and $\gamma_{2}$ shown on Figure 3. As shown on Figure 4, one has $\tau_{\gamma_{1}}^{-1}\left(\gamma_{2}\right)=\beta$ and so, using $T_{1}$ and (1),

$$
a_{\beta}=\overline{a_{\gamma_{1}}} a_{\gamma_{2}} a_{\gamma_{1}}=\overline{a_{\gamma_{1}}} \overline{a_{\gamma_{1}}} a_{\gamma_{1}} a_{\gamma_{2}} a_{\gamma_{1}}=\overline{a_{\gamma_{1}}} \overline{a_{\gamma_{1}}} a_{\gamma_{2}} a_{\gamma_{1}} a_{\gamma_{2}} .
$$

Let us denote by $\delta_{1}, \delta_{2}, \delta_{3}$ and $\delta_{4}$ the curves

$$
\delta_{1}=\tau_{\gamma_{2}}(\alpha), \quad \delta_{2}=\tau_{\gamma_{1}}\left(\delta_{1}\right), \quad \delta_{3}=\tau_{\gamma_{2}}\left(\delta_{2}\right), \quad \delta_{4}=\tau_{\gamma_{1}}^{-1}\left(\delta_{3}\right) .
$$

Then, we have $\gamma=\tau_{\gamma_{1}}^{-1}\left(\delta_{4}\right)$. We will successively show that $\left|\gamma_{2} \cap \alpha\right|,\left|\gamma_{1} \cap \delta_{1}\right|$, $\left|\gamma_{2} \cap \delta_{2}\right|,\left|\gamma_{1} \cap \delta_{3}\right|$ and $\left|\gamma_{1} \cap \delta_{4}\right|$ are smaller than $n-1$. Then, by the induction hypothesis we obtain:

$$
a_{\gamma}=\overline{a_{\gamma_{1}}}\left(a_{\delta_{4}}\right)=\overline{a_{\gamma_{1}}} \overline{a_{\gamma_{1}}}\left(a_{\delta_{3}}\right)=\ldots=\overline{a_{\gamma_{1}}} \overline{a_{\gamma_{1}}} a_{\gamma_{2}} a_{\gamma_{1}} a_{\gamma_{2}}\left(a_{\alpha}\right),
$$

that is to say, $a_{\gamma}=a_{\beta} a_{\alpha} \overline{a_{\beta}}$ using (3).

- By definition, $\left|\gamma_{2} \cap \alpha\right|<n$.

- $\left|\gamma_{1} \cap \delta_{1}\right|=\left|\gamma_{1} \cap \tau_{\gamma_{2}}(\alpha)\right|=\left|\tau_{\gamma_{2}}^{-1}\left(\gamma_{1}\right) \cap \alpha\right|=n-2$ as shown on Figure 5 .

- $\left|\gamma_{2} \cap \delta_{2}\right|=\left|\gamma_{2} \cap \tau_{\gamma_{1}} \tau_{\gamma_{2}}(\alpha)\right|=\left|\tau_{\gamma_{2}}^{-1} \tau_{\gamma_{1}}^{-1}\left(\gamma_{2}\right) \cap \alpha\right|=\left|\tau_{\gamma_{2}}^{-1} \tau_{\gamma_{2}}\left(\gamma_{1}\right) \cap \alpha\right|=\left|\gamma_{1} \cap \alpha\right|<n$. 

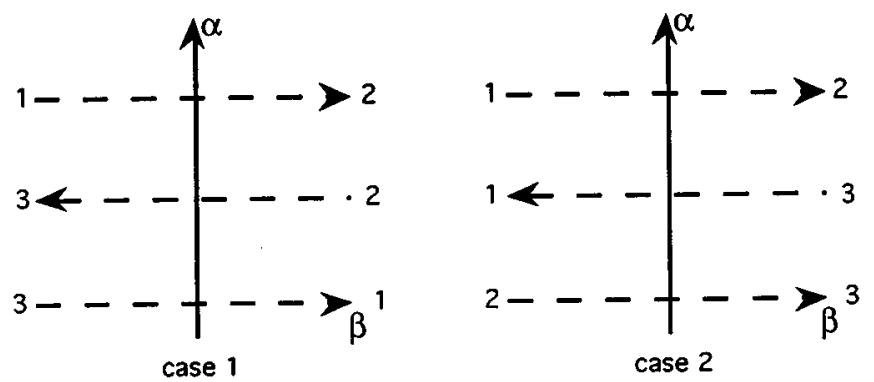

Figure 6
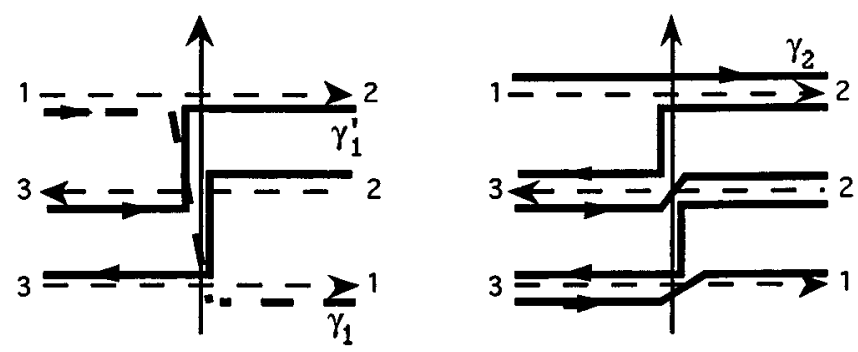

FiguRE 7

- $\left|\gamma_{1} \cap \delta_{3}\right|=\left|\gamma_{1} \cap \tau_{\gamma_{2}} \tau_{\gamma_{1}} \tau_{\gamma_{2}}(\alpha)\right|=\left|\tau_{\gamma_{2}}^{-1} \tau_{\gamma_{1}}^{-1} \tau_{\gamma_{2}}^{-1}\left(\gamma_{1}\right) \cap \alpha\right|=\left|\tau_{\gamma_{2}}^{-1} \tau_{\gamma_{1}}^{-1} \tau_{\gamma_{1}}\left(\gamma_{2}\right) \cap \alpha\right|=$ $\left|\gamma_{2} \cap \alpha\right|<n$.

- $\left|\gamma_{1} \cap \delta_{4}\right|=\left|\tau_{\gamma_{1}}\left(\gamma_{1}\right) \cap \tau_{\gamma_{1}}\left(\delta_{4}\right)\right|=\left|\gamma_{1} \cap \delta_{3}\right|<n$.

Remark. Note that the curves $\gamma_{1}$ and $\gamma_{2}$ do not separate. So, the proof above remains valid for $S$.

Second case. There are not two points in $\alpha \cap \beta$ consecutive on $\alpha$ and with the same intersection sign.

If $n$ is equal to two, then $T_{n}$ is the relation $T_{2_{0}}$ (wich is true in $S$ by Lemma 1.5); so, suppose that $n \geq 3$. As we can see in Figure 6 there are only two different ways to join the strands in order to obtain the simple closed curve $\beta$. Denote by $n_{k}$ the number of points in $\alpha \cap \beta$ which are on the arc $k-k$ of $\beta(k=1,2$ or 3$)$.

Let us consider the first case. If $\gamma_{1}$ and $\gamma_{2}$ are the curves shown in Figure 7 , one has $\left|\gamma_{1} \cap \beta\right|=2_{0}$ and $\tau_{\beta}\left(\gamma_{1}\right)=\gamma_{2}$. So, the relation $T_{2_{0}}$ yields

$$
a_{\gamma_{2}}=a_{\beta} a_{\gamma_{1}} \overline{a_{\beta}}, \quad \text { i.e., } \quad a_{\beta}=\overline{a_{\gamma_{2}}} a_{\beta} a_{\gamma_{1}} .
$$

Now, if $\delta_{1}=\tau_{\gamma_{1}}(\alpha)$ and $\delta_{2}=\tau_{\beta}\left(\delta_{1}\right)$, then $\gamma=\tau_{\gamma_{2}}^{-1}\left(\delta_{2}\right)$.

Since $\left|\gamma_{1} \cap \alpha\right|=n_{1}+1 \leq n-2<n$, one has by the induction hypothesis that $a_{\delta_{1}}=a_{\gamma_{1}}\left(a_{\alpha}\right)$. We have (Figure 8 shows $\tau_{\gamma_{1}}^{-1}(\beta)$ )

$$
\left|\beta \cap \delta_{1}\right|=\left|\beta \cap \tau_{\gamma_{1}}(\alpha)\right|=\left|\tau_{\gamma_{1}}^{-1}(\beta) \cap \alpha\right|=n-2<n
$$

so $a_{\delta_{2}}=a_{\beta}\left(a_{\delta_{1}}\right)=a_{\beta} a_{\gamma_{1}}\left(a_{\alpha}\right)$. Finally,

$$
\left|\gamma_{2} \cap \delta_{2}\right|=\left|\gamma_{2} \cap \tau_{\beta} \tau_{\gamma_{1}}(\alpha)\right|=\left|\tau_{\beta}^{-1}\left(\gamma_{2}\right) \cap \tau_{\gamma_{1}}(\alpha)\right|=\left|\gamma_{1} \cap \tau_{\gamma_{1}}(\alpha)\right|=\left|\gamma_{1} \cap \alpha\right|=n_{1}+1<n
$$




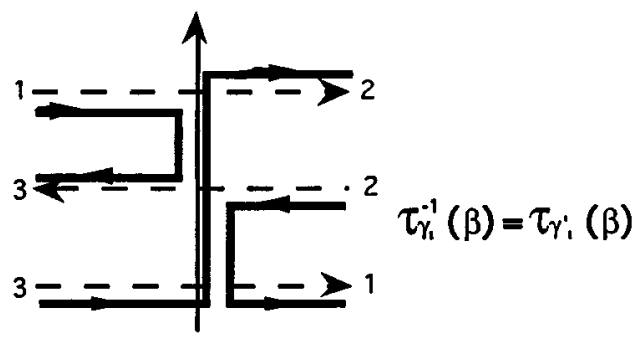

Figure 8
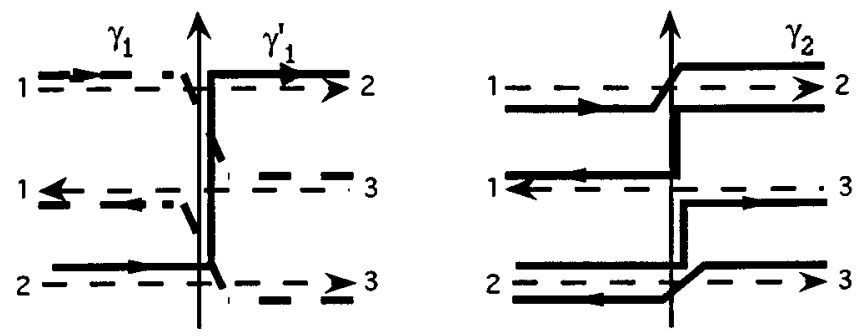

FiguRE 9

and we obtain

$$
a_{\gamma}=\overline{a_{\gamma_{2}}}\left(a_{\delta_{2}}\right)=\overline{a_{\gamma_{2}}} a_{\beta} a_{\gamma_{1}}\left(a_{\alpha}\right)=a_{\beta}\left(a_{\alpha}\right) .
$$

Note that the curve $\gamma_{1}$ (and also $\gamma_{2}$ ) may separate $\Sigma$, and in this case the proof above is incorrect for $S$. But, if $\gamma_{1}^{\prime}$ is the curve shown in Figure 7, then $\left[\gamma_{1}\right]+\left[\gamma_{1}^{\prime}\right]=[\beta]$ (where $[\lambda]$ is the homology class modulo two of the curve $\lambda$ ) and so $\gamma_{1}^{\prime}$ is a non-separating curve if $\beta$ does not separate and $\gamma_{1}$ does.

Let us set $\gamma_{2}^{\prime}=\tau_{\beta}\left(\gamma_{1}^{\prime}\right)$; then, since $\left|\beta \cap \gamma_{1}^{\prime}\right|=2_{0}$, one has by Lemma 1.5

$$
a_{\gamma_{2}^{\prime}}=a_{\beta} a_{\gamma_{1}^{\prime}} \overline{a_{\beta}} \text {, and thus } a_{\beta}=a_{\gamma_{2}^{\prime}} a_{\beta} \overline{a_{\gamma_{1}^{\prime}}} .
$$

If $\delta_{1}^{\prime}$ and $\delta_{2}^{\prime}$ are respectively the curves $\tau_{\gamma_{1}^{\prime}}^{-1}(\alpha)$ and $\tau_{\beta}\left(\delta_{1}^{\prime}\right)$, then $\gamma=\tau_{\gamma_{2}^{\prime}}\left(\delta_{2}^{\prime}\right)$.

- $\left|\gamma_{1}^{\prime} \cap \alpha\right|=2+n_{2}+n_{3}<n$.

- $\left|\beta \cap \delta_{1}^{\prime}\right|=\left|\beta \cap \tau_{\gamma_{1}^{\prime}}^{-1}(\alpha)\right|=\left|\tau_{\gamma_{1}^{\prime}}(\beta) \cap \alpha\right|$.

Since $\tau_{\gamma_{1}^{\prime}}(\beta)=\tau_{\gamma_{1}}^{-1}(\beta)$ (see Figure 8 ), one has $\left|\beta \cap \delta_{1}^{\prime}\right|=n-2$.

- $\left|\gamma_{2}^{\prime} \cap \delta_{2}^{\prime}\right|=\left|\gamma_{2}^{\prime} \cap \tau_{\beta} \tau_{\gamma_{1}^{\prime}}^{-1}(\alpha)\right|=\left|\tau_{\gamma_{1}^{\prime}} \tau_{\beta}^{-1}\left(\gamma_{2}^{\prime}\right) \cap \alpha\right|=\left|\gamma_{1}^{\prime} \cap \alpha\right|<n$.

So, by the induction hypothesis,

$$
a_{\gamma}=a_{\gamma_{2}^{\prime}}\left(a_{\delta_{2}^{\prime}}\right)=a_{\gamma_{2}^{\prime}} a_{\beta}\left(a_{\delta_{1}^{\prime}}\right)=a_{\gamma_{2}^{\prime}} a_{\beta} \overline{a_{\gamma_{1}^{\prime}}}\left(a_{\alpha}\right)=a_{\beta}\left(a_{\alpha}\right) .
$$

For the second case of the Figure 6, the proof is the same using the curves $\gamma_{1}, \gamma_{1}^{\prime}$, and $\gamma_{2}$ of Figure 9

1.2. A relation between the lanterns, the stars and the chains. For any lantern determined by the curves $\beta_{1}$ and $\beta_{3}$, we denote by $L\left(\beta_{1}, \beta_{3}\right)$ the element $a_{\alpha_{1}} a_{\alpha_{2}} a_{\alpha_{3}} a_{\alpha_{4}} \overline{a_{\beta_{1}}} \overline{a_{\beta_{2}}} \overline{a_{\beta_{3}}}$ of $R$, and by $\mathcal{L}$ the subgroup of $R$ generated by all these elements. Similarly, we define elements $E\left(\alpha_{1}, \alpha_{2}, \alpha_{3}, \beta\right)$ and $C\left(\alpha_{1}, \beta, \alpha_{2}\right)$ associated 
to the stars and the chains and the subgroups $\mathcal{E}$ and $\mathcal{C}$. When the curves involved do not separate, we will also consider these elements in $S$, but we will denote them with a subscript " 0 ".

Theorem 1.6. a) (i) The subgroups $\mathcal{L}, \mathcal{E}$ and $\mathcal{C}$ are in the center of $R$.

(ii) If $g \geq 1$, then $\mathcal{E}=\mathcal{C} \mathcal{L}$, where $\mathcal{C} \mathcal{L}$ is the subgroup generated by $\mathcal{C}$ and $\mathcal{L}$.

b) (i) The elements $L_{0}(.,),. E_{0}(., ., .,$.$) and C_{0}(., . .$.$) are central in S$.

(ii) For any lanterns $\left(\beta_{1}, \beta_{3}\right)$ and $\left(\beta_{1}^{\prime}, \beta_{3}^{\prime}\right)$ with non-separating curves, one has in $S$

$$
L_{0}\left(\beta_{1}, \beta_{3}\right)=L_{0}\left(\beta_{1}^{\prime}, \beta_{3}^{\prime}\right)
$$

The same result holds for stars and chains. Let us denote by $L_{0}, E_{0}$ and $C_{0}$ the three elements of $S$ obtained this way.

(iii) $C_{0}=L_{0} E_{0}$ in $S$.

Remark. Part b) makes sense only if the elements $L_{0}, E_{0}$ and $C_{0}$ exists, that is to say, when $g \geq 2$ for $C_{0}$ and when $g \geq 3$ for $L_{0}$ and $E_{0}$.

Proof. The assertion ( $i$ ) of a) and b) is an easy consequence of Corollary 1.4, and (ii) of $\mathrm{b}$ ) is a consequence of $(i)$ and the classification of surfaces. Thus, let us look at the relation $\mathcal{E}=\mathcal{C} \mathcal{L}$. To do this, we first consider a lantern $L=L\left(\beta_{1}, \beta_{3}\right)$ where the curves are denoted as shown in Figure 1.

First case: At least one of the curves $\alpha_{i}$ does not separate. Then, there is a second curve $\alpha_{j}$ which does not separate. Without loss of generality, one can suppose that these two curves are $\alpha_{1}$ and $\alpha_{2}$. Then, if $\beta$ and $\delta$ are the curves shown in Figure 10, one has by Lemma 1.2

$$
C=C\left(\alpha_{1}, \beta, \alpha_{2}\right)=X_{1}^{2} X_{2}^{2} \overline{a_{\delta}} \overline{a_{\beta_{3}}}
$$

and

$$
E=E\left(\alpha_{1}, \beta_{1}, \alpha_{2}, \beta\right)=X_{1} X_{2} X_{3} \overline{a_{\delta}} \overline{a_{\alpha_{4}}} \overline{a_{\alpha_{3}}}
$$

where $X_{1}=a_{\alpha_{1}} a_{\alpha_{2}}, X_{2}=a_{\beta} X_{1} a_{\beta}$ and $X_{3}=a_{\beta_{1}} X_{2} a_{\beta_{1}}$. Thus, we obtain by (0), Lemma 1.2 and $(i)$

$$
C \bar{E}=a_{\alpha_{1}} a_{\alpha_{2}} a_{\alpha_{3}} a_{\alpha_{4}} \overline{a_{\beta_{3}}} \overline{a_{\beta_{1}}} \overline{X_{2}} \overline{a_{\beta_{1}}} X_{2} .
$$

But $\left(\tau_{\beta} \tau_{\alpha_{1}} \tau_{\alpha_{2}} \tau_{\beta}\right)^{-1}\left(\beta_{1}\right)=\beta_{2}$, so, by Corollary 1.4, $a_{\beta_{2}}=\overline{X_{2}}\left(a_{\beta_{1}}\right)$ and we obtain $C \bar{E}=L$. Thus, since $\mathcal{C} \subset \mathcal{E}$, we have $L \in \mathcal{E}$ (by $(i), L=a_{\alpha_{1}} a_{\alpha_{2}} a_{\alpha_{3}} a_{\alpha_{4}} \overline{a_{\beta_{1}}} \overline{a_{\beta_{2}}} \overline{a_{\beta_{3}}}$ $=a_{\alpha_{1}} a_{\alpha_{2}} a_{\alpha_{3}} a_{\alpha_{4}} \overline{a_{\beta_{2}}} \overline{a_{\beta_{3}}} \overline{a_{\beta_{1}}}=a_{\alpha_{1}} a_{\alpha_{2}} a_{\alpha_{3}} a_{\alpha_{4}} \overline{a_{\beta_{3}}} \overline{a_{\beta_{1}}} \overline{a_{\beta_{2}}}$. .

Note that this shows the relation (iii) of b) because if $\beta_{3}$ does not separate, then $\delta$ does not separate either.

Second case: All the curves $\alpha_{i}$ separate in $\Sigma$. Since $g$ is greater than or equal to one, there exists $i$ such that the component of $\Sigma \backslash \alpha_{i}$ which does not contain $\alpha_{j}$ (for all $j \neq i$ ) is of genus greater than or equal to one. We can suppose, without loss of generality, that $i=3$ and consider the curves $\gamma_{1}, \ldots, \gamma_{6}$ shown in Figure 11. 

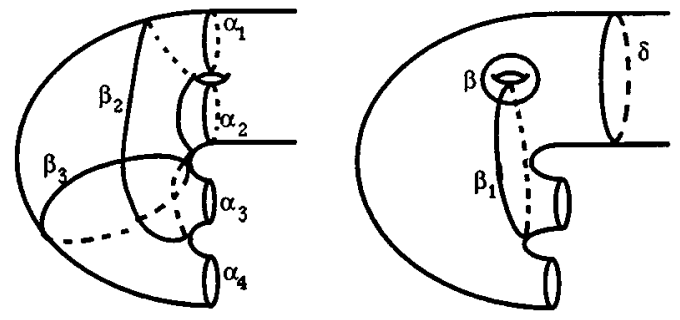

FiguRe 10
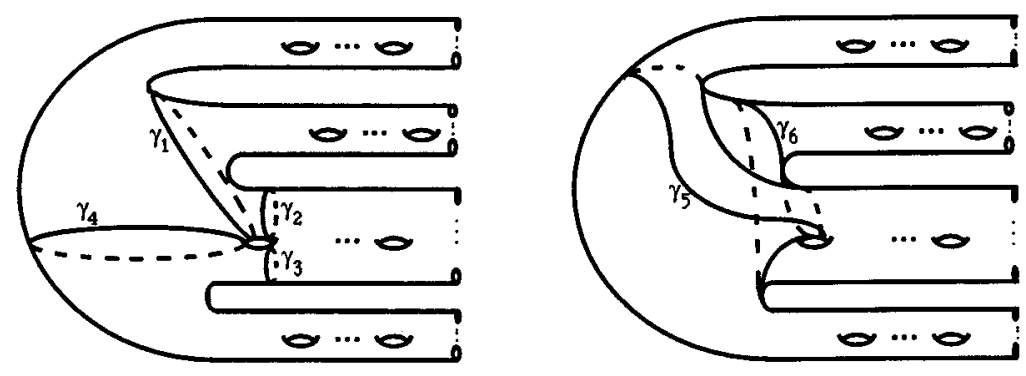

FiguRE 11

Denote respectively by $L_{1}, L_{2}$ and $L_{3}$ the lanterns $L\left(\gamma_{6}, \gamma_{1}\right), L\left(\gamma_{6}, \gamma_{4}\right)$ and $L\left(\gamma_{5}, \gamma_{1}\right)$. Then, using $(0)$ and $(i)$, one has

$$
\begin{aligned}
\overline{L_{1}} L_{2} L_{3}= & \left(a_{\alpha_{2}} a_{\beta_{1}} a_{\gamma_{2}} a_{\gamma_{3}} \overline{a_{\gamma_{6}}} \overline{a_{\alpha_{3}}} \overline{a_{\gamma_{1}}}\right)^{-1}\left(a_{\alpha_{2}} a_{\alpha_{4}} a_{\gamma_{3}} a_{\gamma_{5}} \overline{a_{\gamma_{6}}} \overline{a_{\beta_{2}}} \overline{a_{\gamma_{4}}}\right) \\
& \quad \times\left(a_{\alpha_{1}} a_{\alpha_{2}} a_{\gamma_{2}} a_{\gamma_{4}} \overline{a_{\gamma_{5}}} \overline{a_{\beta_{3}}} \overline{a_{\gamma_{1}}}\right) \\
= & a_{\alpha_{3}} \overline{a_{\gamma_{3}}} \overline{a_{\gamma_{2}}} \overline{a_{\beta_{1}}} a_{\alpha_{4}} a_{\gamma_{3}} \overline{a_{\beta_{2}}} a_{\alpha_{1}} a_{\alpha_{2}} a_{\gamma_{2}} \overline{a_{\beta_{3}}} \\
= & a_{\alpha_{3}} \overline{a_{\beta_{1}}} a_{\alpha_{4}} \overline{a_{\beta_{2}}} a_{\alpha_{1}} a_{\alpha_{2}} \overline{a_{\beta_{3}}} \\
= & L .
\end{aligned}
$$

The first case proves that $L_{k} \in \mathcal{E}$, so we have $L \in \mathcal{E}$.

Thus, we have proved that $\mathcal{L} \subset \mathcal{E}$ and so $\mathcal{L C} \subset \mathcal{E}$. Now, let $\left(\alpha_{1}, \alpha_{2}, \alpha_{3}, \beta\right)$ be a star $E$. We shall find a lantern $L$ and a chain $C$ such that $E=C \bar{L}$ in $R$. Considering the curves shown in Figure 12, one has

$$
\begin{gathered}
E=X_{1} X_{2} X_{3} \overline{a_{\delta_{1}}} \overline{a_{\delta_{2}}} \overline{a_{\delta_{3}}}, \\
C\left(\alpha_{1}, \beta, \alpha_{2}\right)=X_{1}^{2} X_{2}^{2} \overline{a_{\delta_{3}}} \overline{a_{\gamma_{1}}}=C, \\
L\left(\gamma_{2}, \gamma_{1}\right)=a_{\alpha_{1}} a_{\alpha_{2}} a_{\delta_{1}} a_{\delta_{2}} \overline{a_{\alpha_{3}}} \overline{a_{\gamma_{2}}} \overline{a_{\gamma_{1}}}=L,
\end{gathered}
$$

where $X_{1}=a_{\alpha_{1}} a_{\alpha_{2}}, X_{2}=a_{\beta} X_{1} a_{\beta}$ and $X_{3}=a_{\alpha_{3}} X_{2} a_{\alpha_{3}}$. 

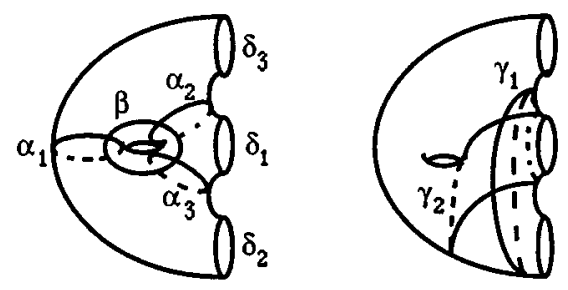

FiguRE 12

Then, using (0) and $(i)$, we obtain

$$
\begin{aligned}
C \bar{L} & =X_{1} X_{2} \overline{a_{\delta_{3}}} X_{1} X_{2} \overline{a_{\gamma_{1}}} a_{\gamma_{1}} a_{\gamma_{2}} a_{\alpha_{3}} \overline{a_{\alpha_{1}}} \overline{a_{\alpha_{2}}} \overline{a_{\delta_{1}}} \overline{a_{\delta_{2}}} \\
& =X_{1} X_{2} \overline{a_{\delta_{3}}} X_{2} a_{\gamma_{2}} a_{\alpha_{3}} \overline{a_{\delta_{1}}} \overline{a_{\delta_{2}}} \\
& =X_{1} X_{2} X_{2} a_{\gamma_{2}} a_{\alpha_{3}} \overline{a_{\delta_{1}}} \overline{a_{\delta_{2}}} \overline{a_{\delta_{3}}} .
\end{aligned}
$$

But one can see that $\tau_{\beta} \tau_{\alpha_{2}} \tau_{\alpha_{1}} \tau_{\beta}\left(\gamma_{2}\right)=\alpha_{3}$, so $a_{\alpha_{3}}=X_{2} a_{\gamma_{2}} \overline{X_{2}}$ by Corollary 1.4. Thus,

$$
\begin{aligned}
C \bar{L} & =X_{1} X_{2} a_{\alpha_{3}} X_{2} a_{\alpha_{3}} \overline{a_{\delta_{1}}} \overline{a_{\delta_{2}}} \overline{a_{\delta_{3}}} \\
& =X_{1} X_{2} X_{3} \overline{a_{\delta_{1}}} \overline{a_{\delta_{2}}} \overline{a_{\delta_{3}}} \\
& =E .
\end{aligned}
$$

This shows that $\mathcal{E} \subset \mathcal{C L}$ and concludes the proof of Theorem 1.6.

We conclude this section by a lemma which gives us a new relation in $\mathcal{M}$.

Lemma 1.7. Let $\Sigma^{\prime}$ be a subsurface of $\Sigma$ which is diffeomorphic to a surface of genus one with four boundary components. Consider the curves shown on Figure 13 and set (in $R$ and $S$ )

$$
X_{1}=a_{\alpha_{1}} a_{\alpha_{3}}, \quad X_{2}=a_{\beta} X_{1} a_{\beta}, \quad X_{3}=a_{\alpha_{2}} X_{2} a_{\alpha_{2}}, \quad X_{3}^{\prime}=a_{\alpha_{4}} X_{2} a_{\alpha_{4}} .
$$

Then, one can find two stars $E_{1}, E_{2}$, and a chain $C$ such that

$$
X_{3} X_{3}^{\prime}=a_{\delta_{1}} a_{\delta_{2}} a_{\delta_{3}} a_{\delta_{4}} E_{1} E_{2} C^{-1} \text { in } R \text {. }
$$

The same result holds in $S$ if $\delta_{1}, \ldots, \delta_{4}$ do not separate in $\Sigma$.

Proof. By Lemma 1.2, one has

$$
\begin{aligned}
E_{1}=E\left(\alpha_{1}, \alpha_{3}, \alpha_{2}, \beta\right) & =X_{1} X_{2} X_{3} \overline{a_{\delta_{2}}} \overline{a_{\delta_{3}}} \overline{a_{\delta}}, \\
E_{2}=E\left(\alpha_{1}, \alpha_{3}, \alpha_{4}, \beta\right) & =X_{1} X_{2} X_{3}^{\prime} \overline{a_{\delta_{1}}} \overline{a_{\delta_{4}}} \overline{a_{\delta^{\prime}}}, \\
C=C\left(\alpha_{1}, \beta, \alpha_{3}\right) & =X_{1}^{2} X_{2}^{2} \overline{a_{\delta}} \overline{a_{\delta^{\prime}}},
\end{aligned}
$$

where $\delta$ and $\delta^{\prime}$ are the curves shown on Figure 14. Thus, we obtain

$$
\begin{aligned}
E_{1} E_{2} C^{-1} & =\overline{a_{\delta_{1}}} \overline{a_{\delta_{2}}} \overline{a_{\delta_{3}}} \overline{a_{\delta_{4}}} X_{1} X_{2} X_{3} \overline{a_{\delta}} X_{1} X_{2} X_{3}^{\prime} \overline{a_{\delta^{\prime}}} a_{\delta} a_{\delta^{\prime}} \overline{X_{1}^{2}} \overline{X_{2}^{2}} \quad \text { by }(0) \\
& =\overline{a_{\delta_{1}}} \overline{a_{\delta_{2}}} \overline{a_{\delta_{3}}} \overline{a_{\delta_{4}}} X_{3} \overline{a_{\delta}} X_{3}^{\prime} a_{\delta} \text { by Lemma } 1.2 \text { and }(0) \\
& =\overline{a_{\delta_{1}}} \overline{a_{\delta_{2}}} \overline{a_{\delta_{3}}} \overline{a_{\delta_{4}}} X_{3} X_{3}^{\prime} \text { by }(0) \text { and }(i) \text { of Theorem } 1.6 .
\end{aligned}
$$

Suppose now that the four curves $\delta_{1}, \ldots, \delta_{4}$ do not separate. The proof above remains valid for $S$ only if both $\delta$ and $\delta^{\prime}$ do not separate $\Sigma$. But if they separate, the curves $\gamma$ and $\gamma^{\prime}$ shown on Figure 14 do not separate. Then, one can conclude in the 


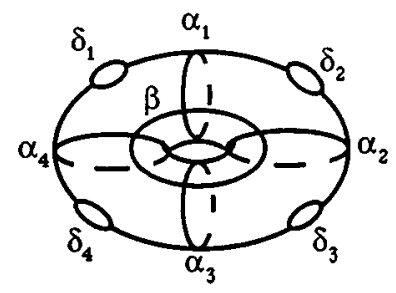

FIGURE 13
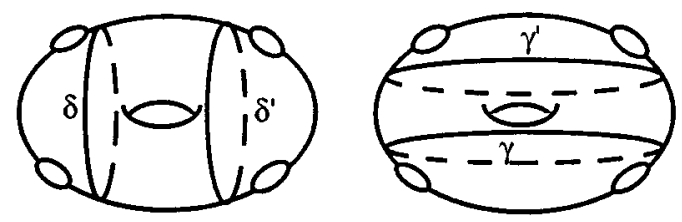

FigURE 14

same way using the stars $\left(\alpha_{2}, \alpha_{4}, \alpha_{1}, \beta\right),\left(\alpha_{2}, \alpha_{4}, \alpha_{3}, \beta\right)$ and the chain $\left(\alpha_{2}, \beta, \alpha_{4}\right)$.

\section{Proof of Theorems A And B}

Let $\Sigma$ be a surface of genus $g \geq 0$ with $r$ boundary components $(r \geq 1), \mathcal{M}=$ $\mathcal{M}_{g, r}$ its mapping class group (we consider $\Sigma$ to be a disc with $g$ handles attached and $r-1$ holes).

In order to simplify notation, we will denote by $\beta$ the twist along a curve $\beta$. First, we shall recall the results obtained by Hatcher-Thurston $([\mathrm{H}-\mathrm{T}])$ and Harer ([H]) about presentations of $\mathcal{M}$. To do this, we will consider the curves shown on Figures 15,16 and 17. If $D_{1}, D_{2}, \ldots, D_{2 g+r-1}$ are the holes obtained when we cut $\Sigma$ along the curves $\alpha_{i}$ or corresponding to $\partial \Sigma$ (exept $\delta$, the boundary of the disc on which the handles were attached), then $\delta_{i j, 1 \leq i<j \leq 2 g+r-1}$ is a curve in $\Sigma \backslash\left(\bigcup \alpha_{i}\right)$ which encircles the holes $D_{i}$, and $D_{j}$ and $\varphi_{k}, 3 \leq k \leq 2 g+r-1$ a curve which encircles $D_{1}, D_{2}$ and $D_{k}$ (see Figure 17).

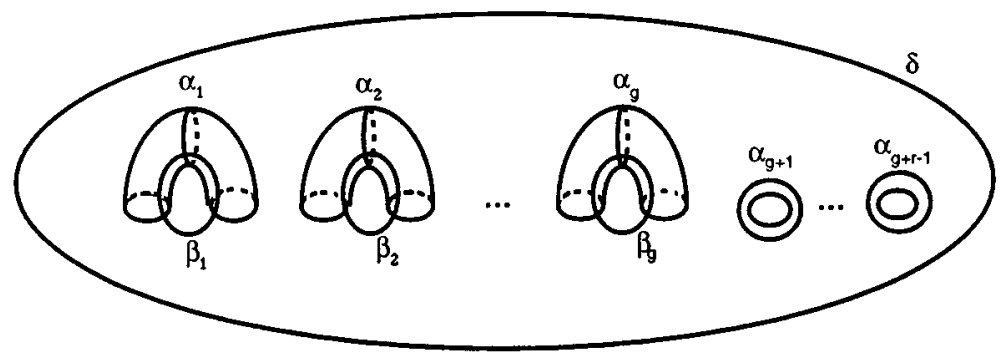

Figure 15 

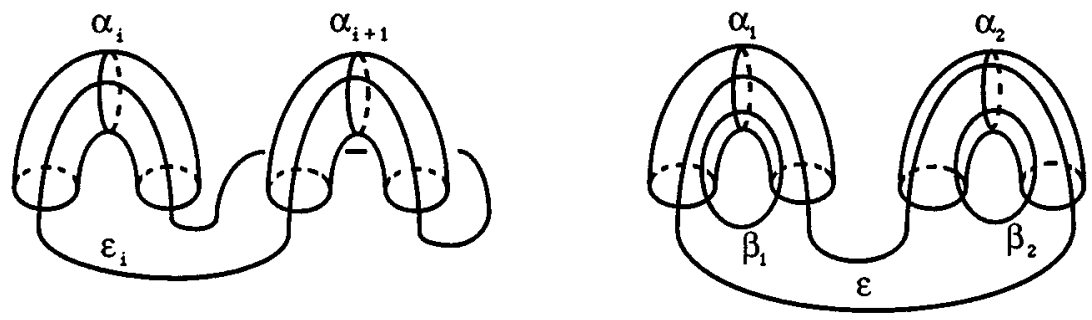

FigURE 16

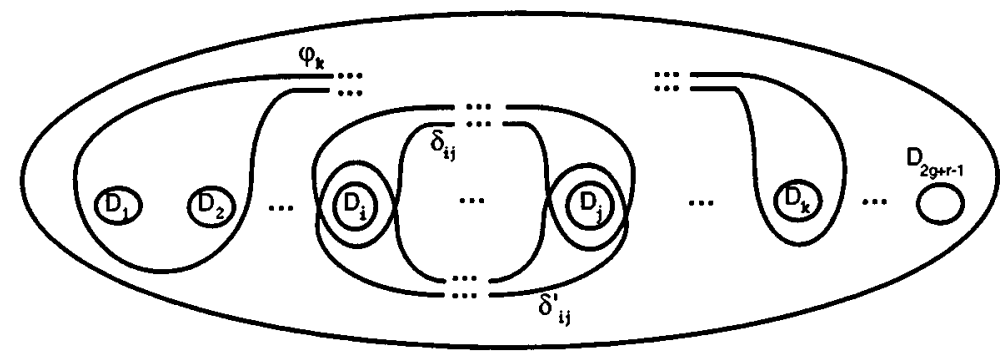

FIGURE 17

Denote by $H_{0}$ the subgroup of elements of $\mathcal{M}$ which leave the curves $\alpha_{i}$ fixed. Hatcher and Thurston have proved in [H-T] that $H_{0}$ is isomorphic to $P_{2 g+r-1} \times$ $\mathbb{Z}^{g+r-1}$, where $P_{n}$ is the pure braid group on $n$ strands. It is generated by $\alpha_{i}$ 's and $\delta_{i, j}$ 's, and admits a presentation with relations (see [B] for a presentation of $\left.P_{n}\right)$

(i) ${ }_{a} \quad \alpha_{i} \alpha_{j}=\alpha_{j} \alpha_{i} \quad \forall i, j \in\langle 1, g+r-1\rangle$,

$(i)_{b} \quad \alpha_{k} \delta_{i j}=\delta_{i j} \alpha_{k} \quad \forall i<j \in\langle 1,2 g+r-1\rangle$ and $k \in\langle 1, g+r-1\rangle$,

$(i)_{c} \delta_{i j} \lambda_{i j k l}=\lambda_{i j k l} \delta_{i j}$, with

$$
\lambda_{i j k l}= \begin{cases}\delta_{k l} & \text { if } 1 \leq k<l<i<j \leq 2 g+r-1 \\ & \text { or } 1 \leq i<k<l<j \leq 2 g+r-1, \\ \delta_{k j} \delta_{k l} & \text { if } 1 \leq k<l=i<j \leq 2 g+r-1, \\ \delta_{l j} \delta_{i j} \delta_{k l} & \text { if } 1 \leq i=k<l<j \leq 2 g+r-1, \\ {\left[\delta_{l j}^{-1}, \delta_{k j}^{-1}\right] \delta_{k l}} & \text { if } 1 \leq k<i<l<j \leq 2 g+r-1 .\end{cases}
$$

$H$ is the subgroup of elements of $\mathcal{M}$ which leave the set $\left\{\alpha_{1}, \ldots, \alpha_{g}\right\}$ fixed (an element of $H$ can permute the $\alpha_{i}$ 's and reverse their orientations). So $H$ is defined by the following exact sequences (see $[\mathrm{W}]$ ):

$$
1 \rightarrow H_{0} \rightarrow H \stackrel{\theta}{\rightarrow} \pm \mathcal{S}_{n} \rightarrow 1 \quad, \quad 1 \rightarrow(\mathbb{Z} / 2 \mathbb{Z})^{n} \rightarrow \pm \mathcal{S}_{n} \rightarrow \mathcal{S}_{n} \rightarrow 1,
$$

where $\mathcal{S}_{n}$ is the symmetric group of rank $n$ and $\theta$ the morphism which assigns to each element of $H$ the induced signed permutation on the $\alpha_{i}$ 's. Therefore, $H$ is generated by $H_{0}, \xi=\beta_{1} \alpha_{1} \alpha_{1} \beta_{1}$ and $\left(\theta_{i}=\varepsilon_{i} \alpha_{i} \alpha_{i+1} \varepsilon_{i}\right)_{1 \leq i \leq g-1}$, and is defined by the relations $(i)$ and 
$(i i)_{a} \theta_{i+1} \theta_{i} \theta_{i+1}=\theta_{i} \theta_{i+1} \theta_{i}$ for $1 \leq i \leq g-2$, and $\theta_{i} \theta_{j}=\theta_{j} \theta_{i}$ for $|i-j| \geq 2$,

$(i i)_{b} \theta_{i}^{2} \in H_{0}$ for $1 \leq i \leq g-1$,

$(i i)_{c} \xi^{2} \in H_{0}$

$(i i)_{d}\left[\xi_{i}, \xi_{j}\right] \in H_{0}$ for $1 \leq i<j \leq g$ where $\xi_{i}=\theta_{i-1} \theta_{i-2} \ldots \theta_{1} \xi \overline{\theta_{1}} \ldots \overline{\theta_{i-2}} \overline{\theta_{i-1}}$,

$(i i)_{e}\left[\theta_{i}, \xi_{j}\right] \in H_{0}$ for $i \neq j, j-1$,

$(\text { iii })_{a} \xi\left(\alpha_{i}\right) \in H_{0}$ for $1 \leq i \leq g+r-1$,

$(i i i)_{b} \xi\left(\delta_{i j}\right) \in H_{0}$ for $1 \leq i<j \leq 2 g+r-1$,

$(\text { iii })_{c} \theta_{k}\left(\alpha_{i}\right) \in H_{0}$ for $1 \leq i \leq g+r-1$ and $1 \leq k \leq g-1$,

$(\text { iii })_{d} \theta_{k}\left(\delta_{i j}\right) \in H_{0}$ for $1 \leq i<j \leq 2 g+r-1$ and $1 \leq k \leq g-1$.

Remark. $\theta_{i}$ permutes $\alpha_{i}$ and $\alpha_{i+1}$, and $\xi_{i}$ reverses the orientation of $\alpha_{i}\left(\xi_{1}=\xi\right)$.

Theorem $2.1([\mathrm{H}-\mathrm{T}][\mathrm{H}]) \cdot \mathcal{M}$ is generated by $H$ and $\sigma=\alpha_{1} \beta_{1} \alpha_{1}$. All relations involving $\sigma$ come from (A)-(E) below and, together with $(i)-($ iii $)$, they suffice for the presentation of $\mathcal{M}$.

(A) $\sigma$ commutes with $H\left(\alpha_{1}, \beta_{1}\right)$, where $H\left(\alpha_{1}, \beta_{1}\right)$ is the subgroup of elements of $H$ which leave $\alpha_{1}$ and $\beta_{1}$ fixed.

(B) $\sigma^{2} \in H$.

(C) Consider the curves $\gamma_{1}, \ldots, \gamma_{N}$ shown on Figure 18. For any $\gamma$ among these curves and any $h \in H$ such that $h \sigma\left(\alpha_{1}\right)=\gamma, h \sigma(\gamma)=\beta_{1}$ and $h \sigma\left(\beta_{1}\right)=\alpha_{1}$, one has $\sigma h \sigma h \sigma \in H$. In the presence of $(\mathrm{A})$, the choice of one $h$ suffices.

(D) For any $h \in H$ such that $h\left(\beta_{2}\right)=\beta_{1}, \sigma$ commutes with $\bar{h}(\sigma)$. In the presence of $(\mathrm{A})$, the choice of one $h$ suffices.

(E) If $\varepsilon$ is the curve shown on Figure 16, then $\sigma h_{5} \sigma h_{4} \sigma h_{3} \sigma h_{2} \sigma h_{1} \sigma \in H$ whenever there are $h_{1}, h_{2}, h_{3}, h_{4}, h_{5} \in H$ such that $h_{1} \sigma\left(\delta_{2,3}\right)=\beta_{1}, h_{2} \sigma h_{1} \sigma(\varepsilon)=$ $\beta_{1}, h_{3} \sigma h_{2} \sigma h_{1} \sigma\left(\alpha_{1}\right)=\beta_{1}, h_{4} \sigma h_{3} \sigma h_{2} \sigma h_{1} \sigma\left(\beta_{2}\right)=\beta_{1}$ and

$$
h_{5} \sigma h_{4} \sigma h_{3} \sigma h_{2} \sigma h_{1} \sigma\left(\alpha_{2}\right)=\beta_{1} \text {. }
$$

The choice of one 5-tuple $\left(h_{1}, \ldots, h_{5}\right) \in H^{5}$ suffices.

Remark. Relations (C) and (E) are slightly different from [H-T]. Moreover, Hatcher and Thurston worked only with closed surfaces. The result above is due to Harer $([\mathrm{H}])$.

Corollary 2.2. Let $\Sigma$ be a surface with at least one boundary component. Then $\mathcal{M}_{\Sigma}$ admits a presentation with generators $\alpha_{i}, \delta_{i, j}, \xi, \theta_{i}, \sigma$ and relations $(i)-($ iii $)$ above added to the following:

(iv) $\sigma$ commutes with $\left(\alpha_{i}\right)_{2 \leq i \leq g+r-1}, \quad\left(\theta_{i}\right)_{2 \leq i \leq g-1}, \quad \xi^{\prime}=\overline{\theta_{1}} \delta_{2,4} \overline{\alpha_{1}}(\xi), \quad \delta_{1,2}$, $\left(\delta_{i j}\right)_{3 \leq i<j \leq 2 g+r-1}$ and $\left(\varphi_{i}\right)_{3 \leq i \leq 2 g+r-1}$, where $\varphi_{i}=\alpha_{1}^{2}\left(\xi \delta_{2, i}\right)^{2} \overline{\alpha_{k_{i}}}$ with $k_{i}=$ $[(i+1) / 2]$ if $i \leq 2 g$ and $k_{i}=i-g$ if $i \geq 2 g+1$.

(v) $\sigma^{2} \in H$.

(vi) $\sigma h \sigma h \sigma \in H \quad$ for all $h \in \mathcal{H}=\left\{\alpha_{1} ; \delta_{1,3} ; \delta_{1,4} \delta_{1,3} \overline{\alpha_{1}} ; \delta_{1,5} \delta_{1,3} \overline{\alpha_{1}} ; \delta_{1,2 g+1}, \ldots\right.$, $\delta_{1,2 g+r-1} ; \delta_{1,2 g+1} \delta_{1,3} \overline{\alpha_{1}}, \ldots, \delta_{1,2 g+r-1} \delta_{1,3} \overline{\alpha_{1}} ; \delta_{1, k} \delta_{1, j} \overline{\alpha_{1}}$ for $2 g+1 \leq$ $j<k \leq 2 g+r-1\}$. 

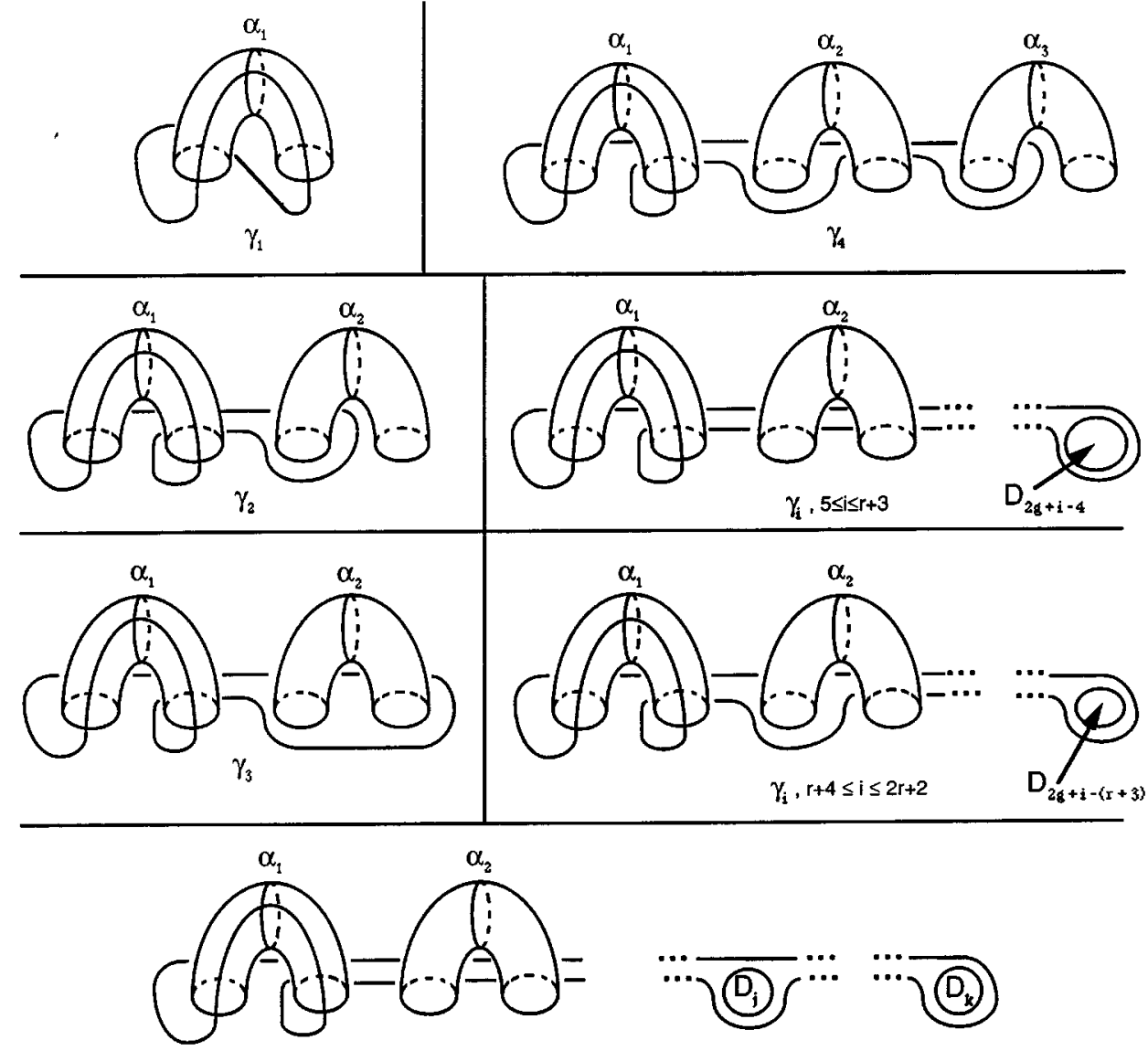

$\gamma_{i}, 2 r+3 \leq i \leq N=2 r+2+C_{r-1}^{2}$

FIGURE 18

(vii) $\sigma$ commutes with $\bar{h} \sigma h$, where $h=\alpha_{1} \overline{\delta_{2,4}} \theta_{1}$.

(viii) $\sigma h_{5} \sigma h_{4} \sigma h_{3} \sigma h_{2} \sigma h_{1} \sigma \in H$, where $h_{1}=h_{3}=h_{5}=\overline{\alpha_{1}} \delta_{2,3}, h_{2}=\overline{\delta_{2,3}} \theta$ and $h_{4}=\delta_{2,3} \overline{\alpha_{1}} \bar{\theta} ;$ here $\theta=\overline{\varphi_{3}} \xi \alpha_{1}^{2} \alpha_{2}\left(\theta_{1}\right)$.

Remarks. (i) $\varphi_{i}$ is the curve shown in Figure 17. The chain $\left(\alpha_{1}, \beta_{1}, \delta_{2, i}\right)$ and the computations of Lemma 1.2 prove that

$$
\varphi_{i}=\left(\alpha_{1} \beta_{1} \delta_{2, i}\right)^{4} \overline{\alpha_{k_{i}}}=\alpha_{1}^{2}\left(\xi \delta_{2, i}\right)^{2} \overline{\alpha_{k_{i}}} .
$$

(ii) As diffeomorphisms of $\Sigma, \xi$ and $\theta$ can be seen to be equal to $\beta_{2} \alpha_{2} \alpha_{2} \beta_{2}$ and $\varepsilon \alpha_{1} \alpha_{2} \varepsilon$ respectively, where the curves are whose which are shown on Figures 15 and 16 .

Proof. We have to prove that the relations (iv)-(viii) are equivalent to the relations (A)-(E).

(iv) Let $H_{0}\left(\alpha_{1}, \beta_{1}\right)$ be the subgroup of elements of $H\left(\alpha_{1}, \beta_{1}\right)$ which leave each curve $\alpha_{i}$ fixed. Then, $H\left(\alpha_{1}, \beta_{1}\right)$ is generated by $H_{0}\left(\alpha_{1}, \beta_{1}\right)$ and elements permuting the $\alpha_{i}$ 's for $2 \leq i \leq g$ or reversing their orientation. We have seen that $\theta_{i}$ permutes $\alpha_{i}$ and $\alpha_{i+1}$. The diffeomorphism $\xi^{\prime}$ preserves $\alpha_{1}, \beta_{1}$ and $\alpha_{i}$ for 
$i \geq 3$ and reverses the orientation of $\alpha_{2}$ (using braid relations, one can see that $\left.\xi^{\prime}=\beta_{2} \alpha_{2} \alpha_{2} \beta_{2}\right)$. So, $H\left(\alpha_{1}, \beta_{1}\right)$ is generated by $H_{0}\left(\alpha_{1}, \beta_{1}\right), \xi^{\prime}$ and $\left(\theta_{i}\right)_{2 \leq i \leq g-1}$. Furthermore, following the proof of Hatcher-Thurston for $H_{0}$, one can prove that $H_{0}\left(\alpha_{1}, \beta_{1}\right)$ is isomorphic to $P_{2 g+r-2} \times \mathbb{Z}^{g+r-2}$ and deduce that it is generated by $\delta_{1,2},\left(\alpha_{i}\right)_{2 \leq i \leq g+r-1},\left(\delta_{i j}\right)_{3 \leq i<j \leq 2 g+r-1}$ and $\left(\varphi_{i}\right)_{3 \leq i \leq 2 g+r-1}$. So, we have found the required generators for $H\left(\alpha_{1}, \beta_{1}\right)$, and this proves that $(i v)$ is equivalent to (A).

(vi) For any s.c.c. $\gamma$ in $\left\{\gamma_{1}, \ldots, \gamma_{N}\right\}$, we have to find an element $h$ of $H$ such that $h \sigma\left(\alpha_{1}\right)=\gamma, h \sigma(\gamma)=\beta_{1}$ and $h \sigma\left(\beta_{1}\right)=\alpha_{1} . \mathcal{H}$ is exactly the set of the elements that we have found:

$$
\begin{aligned}
& * \gamma=\gamma_{1} \Rightarrow h=\alpha_{1}, \\
& * \gamma=\gamma_{2} \Rightarrow h=\delta_{1,3}, \\
& * \gamma=\gamma_{3} \Rightarrow h=\delta_{1,4} \delta_{1,3} \overline{\alpha_{1}}, \\
& * \gamma=\gamma_{4} \Rightarrow h=\delta_{1,5} \delta_{1,3} \overline{\alpha_{1}}, \\
& * \gamma=\gamma_{i}, i=5, \ldots, r+3 \Rightarrow h=\delta_{1,2 g+i-4}, \\
& * \gamma=\gamma_{i}, i=r+4, \ldots, 2 r+2 \Rightarrow h=\delta_{1,2 g+i-r-3} \delta_{1,3} \overline{\alpha_{1}}, \\
& * \gamma=\gamma_{i}, i=2 r+3, \ldots, N \Rightarrow h=\delta_{1, k} \delta_{1, j} \overline{\alpha_{1}} \text { if } \gamma_{i} \text { encircles } D_{j} \text { and } D_{k} .
\end{aligned}
$$

(vii) $h=\alpha_{1} \overline{\delta_{2,4}} \theta_{1}$ is an element of $H$ satisfying $h\left(\beta_{2}\right)=\beta_{1}$, so (vii) is equivalent to $(\mathrm{D})$.

(viii) If we choose $h_{1}=h_{3}=h_{5}=\overline{\alpha_{1}} \delta_{2,3}, h_{2}=\overline{\delta_{2,3}} \theta$ and $h_{4}=\delta_{2,3} \overline{\alpha_{1}} \bar{\theta}$, then $h_{i} \in H$ for all $i$ and the 5 -tuple $\left(h_{1}, h_{2}, h_{3}, h_{4}, h_{5}\right)$ satisfies the condition of the relation $(\mathrm{E})$.

2.1. Proof of Theorem A. Let $G$ be a group with generators $\left\{a \equiv a_{\alpha} / \alpha\right.$ s.c.c. in $\Sigma\}$ and relations (I), (II) and (III) (Theorem A). We shall denote by $H_{0}^{\prime}$ the subgroup of $G$ generated by the $a_{i}$ 's and the $d_{i j}$ 's, and by $H^{\prime}$ the subgroup generated by $H_{0}^{\prime}, x=b_{1} a_{1} a_{1} b_{1}$, and the $t_{i}$ 's, where $t_{i}=e_{i} a_{i} a_{i+1} e_{i} \quad(i=1, \ldots, g-1)$.

Remark. Since $G$ is a quotient of $R$, the relations which have been shown in Section 1 are also true in $G$.

Let $\varphi: G \rightarrow \mathcal{M}$ be the epimorphism defined by $\varphi(a)=\alpha$. In order to prove that $\varphi$ is an isomorphism, we shall construct an inverse morphism $\psi: \mathcal{M} \rightarrow G$. So, let us define $\psi$ on the generators of $\mathcal{M}$ as follows:

$$
\begin{gathered}
\psi(\alpha)=a \quad \forall \alpha \in\left\{\left(\alpha_{i}\right)_{1 \leq i \leq g+r-1} ;\left(\delta_{i j}\right)_{1 \leq i<j \leq 2 g+r-1}\right\}, \\
\psi(\xi)=x, \quad \psi\left(\theta_{i}\right)=t_{i}, \quad \psi(\sigma)=a_{1} b_{1} a_{1}=s .
\end{gathered}
$$

First of all, let us prove that $\psi$ is a homomorphism defined on $\mathcal{M}$; to do this, we will show that the relations (i)-(viii) are mapped by $\psi$ into true relations in $G$.

Relations $(\boldsymbol{i}) .(i)_{a}$ and $(i)_{b}$ are consequences of $(0)$, and since $\lambda_{i j k l}\left(\delta_{i j}\right)=\delta_{i j}$, $(i)_{c}$ is a consequence of Corollary 1.4 .

Relation (ii) $a \cdot(0)$ implies $t_{i} t_{j}=t_{j} t_{i}$ when $|i-j| \geq 2$. For $1 \leq i \leq g-1$, one has 


$$
\begin{aligned}
t_{i+1}\left(t_{i}\right) & =\left(e_{i+1} a_{i+1} a_{i+2} e_{i+1}\right)\left(e_{i} a_{i} a_{i+1} e_{i}\right)\left(\overline{e_{i+1}} \overline{a_{i+1}} \overline{a_{i+2}} \overline{e_{i+1}}\right) \\
& =e_{i+1} a_{i+1} a_{i+2} e_{i} a_{i} e_{i+1} a_{i+1} \overline{e_{i+1}} e_{i} \overline{a_{i+1}} \overline{a_{i+2}} \overline{e_{i+1}} \quad \text { by }(0) \\
& =e_{i+1} a_{i+1} a_{i+2} e_{i} a_{i} \overline{a_{i+1}} e_{i+1} a_{i+1} e_{i} \overline{a_{i+1}} \overline{a_{i+2}} \overline{e_{i+1}} \quad \text { by }(1) \\
& =e_{i+1} a_{i+2} a_{i+1} e_{i} \overline{a_{i+1}} a_{i} e_{i+1} a_{i+1} e_{i} \overline{a_{i+1}} \overline{a_{i+2}} \overline{e_{i+1}} \text { by }(0) \\
& =e_{i+1} a_{i+2} \overline{e_{i}} a_{i+1} e_{i} a_{i} e_{i+1} \overline{e_{i}} a_{i+1} e_{i} \overline{a_{i+2}} \overline{e_{i+1}} \text { by }(1) \\
& =\overline{e_{i}} e_{i+1} a_{i+2} a_{i+1} e_{i} a_{i} \overline{e_{i}} e_{i+1} a_{i+1} \overline{a_{i+2}} \overline{e_{i+1}} e_{i} \text { by }(0) \\
& =\overline{e_{i}} e_{i+1} a_{i+2} a_{i+1} \overline{a_{i}} e_{i} a_{i} e_{i+1} a_{i+1} \overline{a_{i+2}} \overline{e_{i+1}} e_{i} \text { by }(1) \\
& =\overline{e_{i}} \overline{a_{i}} e_{i+1} a_{i+1} e_{i} a_{i+2} e_{i+1} \overline{a_{i+2}} a_{i+1} \overline{e_{i+1}} a_{i} e_{i} \text { by }(0) \\
& =\overline{e_{i}} \overline{a_{i}} e_{i+1} a_{i+1} e_{i} \overline{e_{i+1}} a_{i+2} e_{i+1} a_{i+1} \overline{e_{i+1}} a_{i} e_{i} \text { by }(1) \\
& =\overline{e_{i}} \overline{a_{i}} e_{i+1} a_{i+1} \overline{e_{i+1}} e_{i} a_{i+2} e_{i+1} a_{i+1} \overline{e_{i+1}} a_{i} e_{i} \text { by }(0) \\
& =\overline{e_{i}} \overline{a_{i}} \overline{a_{i+1}} e_{i+1} a_{i+1} e_{i} a_{i+2} \overline{a_{i+1}} e_{i+1} a_{i+1} a_{i} e_{i} \quad \text { by }(1) \\
& =\overline{e_{i}} \overline{a_{i}} \overline{a_{i+1}} e_{i+1} a_{i+1} e_{i} \overline{a_{i+1}} a_{i+2} e_{i+1} a_{i+1} a_{i} e_{i} \quad \text { by }(0) \\
& =\overline{e_{i}} \overline{a_{i}} \overline{a_{i+1}} e_{i+1} \overline{e_{i}} a_{i+1} e_{i} a_{i+2} e_{i+1} a_{i+1} a_{i} e_{i} \text { by }(1) \\
& =\overline{e_{i}} \overline{a_{i}} \overline{a_{i+1}} \overline{e_{i}} e_{i+1} a_{i+1} a_{i+2} e_{i+1} e_{i} a_{i+1} a_{i} e_{i} \quad \text { by }(0) \\
& =\overline{t_{i}}\left(t_{i+1}\right)
\end{aligned}
$$

which implies $t_{i+1} t_{i} t_{1+1}=t_{i} t_{i+1} t_{i}$.

Relation $(\boldsymbol{i i})_{\boldsymbol{b}}$. Let $\delta_{i j}^{\prime}$ be the curves shown in Figure 17.

Lemma 2.3. $d_{i j}^{\prime} \in H_{0}^{\prime}$ for all $1 \leq i<j \leq 2 g+r-1$, and $H_{0}^{\prime}$ is generated by $a_{i}$ 's and $d_{i j}^{\prime}$ 's.

Proof. Since $\delta_{i, j}=\tau_{\delta_{i, j-1}} \ldots \tau_{\delta_{i, i+1}}\left(\delta_{i, j}^{\prime}\right)$, one has

$$
d_{i, j}^{\prime}=\overline{d_{i, i+1}} \ldots \overline{d_{i, j-1}}\left(d_{i, j}\right) \in H_{0}^{\prime}
$$

(use Corollary 1.4). Symmetrically, we obtain $d_{i, j}=d_{i, i+1}^{\prime} \ldots d_{i, j-1}^{\prime}\left(d_{i, j}^{\prime}\right)$, which proves the lemma.

By Lemma 1.2, one has $\left(a_{i} e_{i} a_{i+1}\right)^{4}=a_{i}^{2} a_{i+1}^{2} t_{i}^{2}$. But the chain $\left(\alpha_{i}, \varepsilon_{i}, \alpha_{i+1}\right)$ gives:

$$
\left(a_{i} e_{i} a_{i+1}\right)^{4}=d_{2 i-1,2 i+1}^{\prime} d_{2 i, 2 i+2} .
$$

Thus, by Lemma $2.3, t_{i}^{2}$ is an element of $H_{0}^{\prime}$.

Relation $(\boldsymbol{i i}) \boldsymbol{c}$. The chain $\left(\alpha_{1}, \beta_{1}, \alpha_{1}\right)$ yields the relation $a_{1}^{4}\left(b_{1} a_{1} a_{1} b_{1}\right)^{2}=d_{1,2}$ (using Lemma 1.2). Thus we have $x^{2}=d_{1,2} a_{1}^{-4} \in H_{0}^{\prime}$.

Relation (iii) $\boldsymbol{a}$. For $i \geq 2, x\left(a_{i}\right)=a_{i} \in H_{0}^{\prime}$ by (0). One has

$$
x\left(a_{1}\right)=b_{1} a_{1} a_{1} b_{1}\left(a_{1}\right)=b_{1} a_{1} a_{1} \overline{a_{1}}\left(b_{1}\right)=b_{1} \overline{b_{1}}\left(a_{1}\right)=a_{1} \in H_{0}^{\prime} \quad \text { by }(1) .
$$

Relation $\left({ }_{i i i}\right)_{\boldsymbol{b}}$. When $3 \leq i<j \leq 2 g+r-1$ or $(i, j)=(1,2)$, one has $x\left(d_{i, j}\right)=d_{i, j} \in H_{0}^{\prime}$ by $(0)$.

When $3 \leq j \leq 2 g+r-1$, one has $\tau_{\delta_{1,2}}^{-1} \xi\left(\delta_{1, j}\right)=\delta_{2, j}$ so, according to Corollary 1.4, $x\left(d_{1, j}\right)=d_{1,2}\left(d_{2, j}\right) \in H_{0}^{\prime}$.

Similarly, when $3 \leq j \leq 2 g+r-1$, we have $x\left(d_{2, j}\right)=d_{1, j} \in H_{0}^{\prime}$.

Relation $(\boldsymbol{i i i}) \boldsymbol{c}$. We have seen that $\theta_{k}$ permutes $\alpha_{k}$ and $\alpha_{k+1}$ and leaves $\alpha_{i}$ fixed when $i \neq k, k+1$. So, by Corollary 1.4, one has $t_{k}\left(a_{i}\right) \in H_{0}^{\prime}$. 
Relation $(\boldsymbol{i i i})_{\boldsymbol{d}}$. For all $i, j, k$, we shall find $h \in H_{0}$ such that $\theta_{k}\left(\delta_{i j}\right)=$ $h\left(\delta_{i^{\prime}, j^{\prime}}\right)$ for some $i^{\prime}, j^{\prime}$. Then, if $h$ is given as a word on the generators $a_{i}$ 's and $\delta_{i, j}$ 's, and $m$ is the associated word in $H_{0}^{\prime}$, one has by Corollary 1.4

$$
t_{k}\left(d_{i j}\right)=m\left(d_{i^{\prime}, j^{\prime}}\right) \in H_{0}^{\prime} .
$$

The cases of $\delta_{2 k-1,2 k+1}, \delta_{2 k+1, j}$ and $\delta_{i, 2 k+1}$ will be proved in a slightly different way:

$$
\begin{aligned}
& * i, j \neq 2 k-1,2 k, 2 k+1,2 k+2 \Rightarrow \theta_{k}\left(\delta_{i, j}\right)=\delta_{i, j} . \\
& * j \geq 2 k+3 \Rightarrow \theta_{k}\left(\delta_{2 k-1, j}\right)=\delta_{2 k-1,2 k+1}^{\prime} \delta_{2 k, 2 k+1}^{\prime}\left(\delta_{2 k+1, j}\right) . \\
& \quad i \leq 2 k-2 \Rightarrow \theta_{k}\left(\delta_{i, 2 k-1}\right)=\delta_{2 k-1,2 k+1}^{\prime} \delta_{2 k, 2 k+1}^{\prime}\left(\delta_{i, 2 k+1}\right) . \\
& * j \geq 2 k+3 \Rightarrow \theta_{k}\left(\delta_{2 k, j}\right)=\delta_{2 k, 2 k+2}\left(\delta_{2 k+2, j}\right) . \\
& \quad i \leq 2 k-2 \Rightarrow \theta_{k}\left(\delta_{i, 2 k}\right)=\delta_{2 k, 2 k+2}\left(\delta_{i, 2 k+2}\right) . \\
& * j \geq 2 k+3 \Rightarrow \theta_{k}\left(\delta_{2 k+2, j}\right)=\delta_{2 k+1, j} . \\
& \quad i \leq 2 k-2 \Rightarrow \theta_{k}\left(\delta_{i, 2 k+2}\right)=\delta_{i, 2 k+1} . \\
& * \theta_{k}\left(\delta_{2 k-1,2 k}\right)=\delta_{2 k-1,2 k+1}^{\prime}\left(\delta_{2 k+1,2 k+2}\right) . \\
& * \theta_{k}\left(\delta_{2 k-1,2 k+2}\right)=\delta_{2 k-1,2 k}^{-1}\left(\delta_{2 k, 2 k+1}\right) . \\
& * \theta_{k}\left(\delta_{2 k, 2 k+1}\right)=\delta_{2 k+1,2 k+2}^{-1}\left(\delta_{2 k-1,2 k+2}^{\prime}\right) . \\
& * \theta_{k}\left(\delta_{2 k, 2 k+2}\right)=\delta_{2 k, 2 k+2} \\
& * \theta_{k}\left(\delta_{2 k+1,2 k+2}\right)=\delta_{2 k, 2 k+2}\left(\delta_{2 k-1,2 k}\right) . \\
& * \text { Since } \delta_{2 k-1,2 k+1}=\delta_{2 k-1,2 k}\left(\delta_{2 k-1,2 k+1}^{\prime}\right), \text { one has } \\
& \theta_{k}\left(\delta_{2 k-1,2 k+1}\right)=\theta_{k} \delta_{2 k-1,2 k}\left(\delta_{2 k-1,2 k+1}^{\prime}\right)=\theta_{k} \delta_{2 k-1,2 k} \overline{\theta_{k}} \theta_{k}\left(\delta_{2 k-1,2 k+1}^{\prime}\right) .
\end{aligned}
$$

But since

$$
\theta_{k}\left(\delta_{2 k-1,2 k+1}^{\prime}\right)=\delta_{2 k-1,2 k+1}^{\prime},
$$

one has using Corollary 1.4

$$
t_{k}\left(d_{2 k-1,2 k+1}\right)=t_{k} d_{2 k-1,2 k} \overline{t_{k}}\left(d_{2 k-1,2 k+1}^{\prime}\right) .
$$

We have seen that $t_{k}\left(d_{2 k-1,2 k}\right) \in H_{0}^{\prime}$; therefore $t_{k}\left(d_{2 k-1,2 k+1}\right) \in H_{0}^{\prime}$ by Lemma 2.3.

* We saw in Lemma 2.3 that

$$
d_{2 k+1, j}=d_{2 k+1, j-1} \ldots d_{2 k+1,2 k+2}\left(d_{2 k+1, j}^{\prime}\right) .
$$

Since, for $j \geq 2 k+3, \theta_{k}\left(\delta_{2 k+1, j}^{\prime}\right)=\delta_{2 k-1,2 k+1}^{\prime}\left(\delta_{2 k-1, j}^{\prime}\right)$, one has $t_{k}\left(d_{2 k+1, j}^{\prime}\right)$ $\in H_{0}^{\prime}$ by Corollary 1.4. Furthermore, we already have $t_{k}\left(d_{2 k+1,2 k+2}\right) \in$ $H_{0}^{\prime}$, thus, $t_{k}\left(d_{2 k+1, j}\right) \in H_{0}^{\prime}$ by induction on $j$.

* Similarly, one has $d_{i, 2 k+1}=d_{i, 2 k} \ldots d_{i, i+1}\left(d_{i, 2 k+1}^{\prime}\right)$ and, for $i$ less than $2 k-1, t_{k}\left(d_{i, 2 k+1}^{\prime}\right)=d_{2 k-1,2 k+1}^{\prime}\left(d_{i, 2 k-1}^{\prime}\right) \in H_{0}^{\prime}$. Since $t_{k}\left(d_{i, l}\right) \in H_{0}^{\prime}$ for $l=i+1, \ldots, 2 k$, we have $t_{k}\left(d_{i, 2 k+1}\right) \in H_{0}^{\prime}$.

\section{Relation $(i i)_{d}$.}

Lemma 2.4. $t_{1}$ commutes with $x t_{1} x$ in $G$.

Proof. We have 


$$
\begin{aligned}
& x t_{1} x t_{1}=\left(b_{1} a_{1} a_{1} b_{1}\right)\left(e_{1} a_{1} a_{2} e_{1}\right)\left(b_{1} a_{1} a_{1} b_{1}\right)\left(e_{1} a_{1} a_{2} e_{1}\right) \\
& =b_{1} a_{1} a_{1} e_{1} b_{1} a_{1} b_{1} a_{2} e_{1} a_{1} a_{1} b_{1} e_{1} a_{1} a_{2} e_{1} \text { by }(0) \\
& =b_{1} a_{1} a_{1} e_{1} a_{1} b_{1} a_{1} a_{2} e_{1} a_{1} a_{1} b_{1} e_{1} a_{1} a_{2} e_{1} \text { by } \quad(1) \\
& =b_{1} a_{1} e_{1} a_{1} e_{1} b_{1} a_{2} a_{1} e_{1} a_{1} a_{1} e_{1} b_{1} a_{1} a_{2} e_{1} \text { by (0) and (1) } \\
& =b_{1} e_{1} a_{1} e_{1} b_{1} e_{1} a_{2} e_{1} a_{1} e_{1} a_{1} e_{1} b_{1} a_{1} a_{2} e_{1} \text { by (0) and (1) } \\
& =e_{1} b_{1} a_{1} b_{1} e_{1} e_{1} a_{2} e_{1} a_{1} a_{1} e_{1} a_{1} b_{1} a_{1} a_{2} e_{1} \text { by (0) and (1) } \\
& =e_{1} a_{1} b_{1} a_{1} e_{1} a_{2} e_{1} a_{2} a_{1} a_{1} e_{1} b_{1} a_{1} b_{1} a_{2} e_{1} \text { by (1) } \\
& =e_{1} a_{1} b_{1} a_{1} a_{2} e_{1} a_{2} a_{1} a_{1} a_{2} e_{1} a_{2} b_{1} a_{1} e_{1} b_{1} \text { by (0) and (1) } \\
& =e_{1} a_{1} a_{2} b_{1} a_{1} e_{1} a_{1} a_{2} a_{1} b_{1} e_{1} a_{2} e_{1} a_{1} e_{1} b_{1} \text { by (0) and (1) } \\
& =e_{1} a_{1} a_{2} b_{1} e_{1} a_{1} e_{1} a_{1} b_{1} a_{2} e_{1} a_{2} a_{1} e_{1} a_{1} b_{1} \text { by (0) and (1) } \\
& =e_{1} a_{1} a_{2} e_{1} b_{1} a_{1} e_{1} a_{1} b_{1} e_{1} a_{2} e_{1} a_{1} e_{1} a_{1} b_{1} \text { by (0) and (1) } \\
& =e_{1} a_{1} a_{2} e_{1} b_{1} a_{1} e_{1} a_{1} e_{1} b_{1} a_{2} a_{1} e_{1} a_{1} a_{1} b_{1} \text { by (0) and (1) } \\
& =e_{1} a_{1} a_{2} e_{1} b_{1} a_{1} a_{1} e_{1} a_{1} b_{1} a_{1} a_{2} e_{1} a_{1} a_{1} b_{1} \text { by (0) and (1) } \\
& =e_{1} a_{1} a_{2} e_{1} b_{1} a_{1} a_{1} e_{1} b_{1} a_{1} b_{1} a_{2} e_{1} a_{1} a_{1} b_{1} \text { by (1) } \\
& =e_{1} a_{1} a_{2} e_{1} b_{1} a_{1} a_{1} b_{1} e_{1} a_{1} a_{2} e_{1} b_{1} a_{1} a_{1} b_{1} \text { by } \quad(0) \\
& =t_{1} x t_{1} x \text {. }
\end{aligned}
$$

Denote $\psi\left(\xi_{i}\right)=t_{i-1} t_{i-2} \ldots t_{1}(x)$ by $x_{i}$; we have to prove that $\left[x_{i}, x_{j}\right] \in H_{0}^{\prime} \quad(i<$ $j$ ). Relations $(\mathrm{iii})_{c-d}$ show that $H_{0}^{\prime}$ is stable under conjugation by $t_{i}$. So, one can conjugate by $g=\overline{t_{2}} \ldots \overline{t_{i}} \overline{t_{1}} \ldots \overline{t_{j-1}}$ :

$$
g t_{j-1} \ldots t_{1}(x)=\overline{t_{2}} \ldots \overline{t_{i}}(x)=x \quad \text { by }(0),
$$

and

$$
\begin{aligned}
g t_{i-1} \ldots t_{1}(x) & =\overline{t_{2}} \ldots \overline{t_{i}} \overline{t_{1}} \ldots \overline{t_{i-1}} \overline{t_{i}} \ldots \overline{t_{j-1}} t_{i-1} \ldots t_{1}(x) \\
& =\overline{t_{2}} \ldots \overline{t_{i}} \overline{t_{1}} \ldots \overline{t_{i-1}} \overline{t_{i}} t_{i-1} \ldots t_{1}(x) \quad \text { by }(i i)_{a} \text { and }(0) \\
& =\overline{t_{2}} \ldots \overline{t_{i}} \overline{t_{1}} \ldots \overline{t_{i-2}} t_{i} \overline{t_{i-1}} \overline{t_{i}} t_{i-2} \ldots t_{1}(x) \quad \text { by }(i i)_{a} \\
& =\overline{t_{2}} \ldots \overline{t_{i}} t_{i} \overline{t_{1}} \ldots \overline{t_{i-2}} \overline{t_{i-1}} t_{i-2} \ldots t_{1}(x) \quad \text { by }(i i)_{a} \text { and }(0) \\
& =\overline{t_{2}} \ldots \overline{t_{i-1}} \overline{t_{1}} \ldots \overline{t_{i-3}} t_{i-1} \overline{t_{i-2}} \overline{t_{i-1}} t_{i-3} \ldots t_{1}(x) \quad \text { by }(i i)_{a} \\
& \vdots \\
& =\overline{t_{2}} \overline{t_{1}} \overline{t_{2}} t_{1}(x) \\
& =\overline{t_{2}} t_{2} \overline{t_{1}} \overline{t_{2}}(x) \quad \text { by }(i i)_{a} \\
& =\overline{t_{1}}(x) \text { by (0). }
\end{aligned}
$$

Thus, $(i i)_{d}$ is equivalent to $\left[\overline{t_{1}}(x), x\right] \in H_{0}^{\prime}$. But, by Lemma 2.4 , we have

$$
\left[\overline{t_{1}}(x), x\right]=\overline{t_{1}} x t_{1} x \overline{t_{1}} \bar{x} t_{1} \bar{x}={\overline{t_{1}}}^{2} x t_{1}^{2} \bar{x} .
$$

Then, relations $(i i)_{b}$ and $(i i i)_{a-b}$ yield $(i i)_{d}$.

Relation $(\boldsymbol{i i}) \boldsymbol{e}$. For $i \geq j+1$, relations $(i i)_{a}$ and (0) give

$$
t_{i}\left(x_{j}\right)=t_{i} t_{j-1} \ldots t_{1}(x)=t_{j-1} \ldots t_{1} t_{i}(x)=x_{j}
$$

i.e.

$$
\left.t_{i}, x_{j}\right]=1 \in H_{0}^{\prime}
$$


For $i \leq j-2$, the same relations give

$$
\begin{aligned}
t_{i}\left(x_{j}\right) & =t_{i} t_{j-1} \ldots t_{1}(x) \\
& =t_{j-1} \ldots t_{i} t_{i+1} t_{i} \ldots t_{1}(x) \\
& =t_{j-1} \ldots t_{i+1} t_{i} t_{i+1} \ldots t_{1}(x) \\
& =t_{j-1} \ldots t_{i+1} t_{i} \ldots t_{1} t_{i+1}(x) \\
& =t_{j-1} \ldots t_{i+1} t_{i} \ldots t_{1}(x)=x_{j} .
\end{aligned}
$$

Thus $\left[t_{i}, x_{j}\right]=1 \in H_{0}^{\prime}$.

Relation (iv). The relation (0) shows that $s$ commutes with $\left(a_{i}\right)_{2 \leq i \leq g+r-1}$, $\left(t_{i}\right)_{2 \leq i \leq g-1}, d_{1,2},\left(d_{i j}\right)_{3 \leq i<j \leq 2 g+r-1}$.

By Corollary 1.4, one has $\psi\left(\xi^{\prime}\right)=\overline{t_{1}} d_{2,4} \overline{a_{1}}(x)=b_{2} a_{2} a_{2} b_{2}$ and so (0) implies that $s$ commutes with $\psi\left(\xi^{\prime}\right)$.

Chain relations and Lemma 1.2 show that $\psi\left(\varphi_{i}\right)=a_{1}^{2}\left(x d_{2, i}\right)^{2} \overline{a_{k_{i}}}$ is equal to $f_{i}$. Thus, $s$ commutes with $\psi\left(\varphi_{i}\right)$ by $(0)$.

Relation $(\boldsymbol{v}) . s^{2}=a_{1} x a_{1} \in H^{\prime}$.

Relation (vi). Suppose first that $m=\psi(h) \in\left\{a_{1} ; d_{1,3} ; d_{1,2 g+1}, \ldots, d_{1,2 g+r-1}\right\}$. Then

So,

$$
m a_{1}=a_{1} m \text { by }(0) \quad \text { and } \quad b_{1} m b_{1}=m b_{1} m \text { by }(1) \text {. }
$$

$$
\begin{aligned}
s m s m s & =a_{1} b_{1} a_{1} m a_{1} b_{1} a_{1} m a_{1} b_{1} a_{1}=a_{1} b_{1} a_{1} a_{1} m b_{1} m a_{1} a_{1} b_{1} a_{1} \\
& =a_{1} x m x a_{1} \in H^{\prime} .
\end{aligned}
$$

Suppose next that

$$
m \in\left\{d_{1,4} d_{1,3} \overline{a_{1}} ; d_{1,5} d_{1,3} \overline{a_{1}} ; d_{1,2 g+1} d_{1,3} \overline{a_{1}}, \ldots, d_{1,2 g+r-1} d_{1,3} \overline{a_{1}} ; d_{1, k} d_{1, j} \overline{a_{1}}\right.
$$

for $2 g+1 \leq j<k \leq 2 g+r-1\}$;

$m$ can be seen as a product $d_{1, k} d_{1, j} \overline{a_{1}}$ with $j=3$ and $k \in\{4,5,2 g+1, \ldots$, $2 g+r-1\}$ or $2 g+1 \leq j<k \leq 2 g+r-1$.

If $\mu_{j k}$ is a curve in $\Sigma \backslash\left\{\alpha_{i}\right\} \subset \Sigma$ which encircles $D_{1}, D_{j}$ and $D_{k}$, the lantern $L\left(\delta_{j, k}, \delta_{1, k}\right)$ yields

$$
a_{1} a_{i_{j}} a_{i_{k}} m_{j k}=d_{1, k} d_{1, j} d_{j k} .
$$

Thus, $m=a_{i_{j}} a_{i_{k}} m_{j k} \overline{d_{j k}}$ and

$$
\begin{aligned}
\text { smsms } & =\left(a_{1} b_{1} a_{1}\right)\left(\overline{d_{j k}} a_{i_{j}} a_{i_{k}} m_{j k}\right)\left(a_{1} b_{1} a_{1}\right)\left(\overline{d_{j k}} a_{i_{j}} a_{i_{k}} m_{j k}\right)\left(a_{1} b_{1} a_{1}\right) \\
& ={\overline{d_{j k}}}^{2} a_{i_{j}}^{2} a_{i_{k}}^{2} a_{1} b_{1} a_{1} a_{1} m_{j k} b_{1} m_{j k} a_{1} a_{1} b_{1} a_{1} \quad \text { by }(0) \\
& =\bar{d}_{j k}^{2} a_{i_{j}}^{2} a_{i_{k}}^{2} a_{1} x m_{j k} x a_{1} \quad \text { by }(1) .
\end{aligned}
$$

It is already known that $a_{1}, a_{i_{j}}, a_{i_{k}}, x$ and $d_{j k}$ are elements of $H^{\prime} ;(4)$ shows that $m_{j k} \in H^{\prime}$. Therefore, smsms $\in H^{\prime}$.

Relation (vii). If $h=\tau_{\alpha_{1}} \tau_{\delta_{2,4}}^{-1} \tau_{\varepsilon_{1}} \tau_{\alpha_{1}} \tau_{\alpha_{2}} \tau_{\varepsilon_{1}}$, then $h^{-1}\left(\alpha_{1}\right)=\alpha_{2}$ and $h^{-1}\left(\beta_{1}\right)=$ $\beta_{2}$. Thus, by Corollary 1.4, one has $\bar{m}\left(a_{1}\right)=a_{2}$ and $\bar{m}\left(b_{1}\right)=b_{2}$, where $m=\psi(h)=$ $a_{1} d_{2,4} t_{1}$, so $\bar{m}(s)=a_{2} b_{2} a_{2}$. Then, $s$ commutes with $\bar{m}(s)$ by $(0)$.

Relation (viii). Set $m_{i}=\psi\left(h_{i}\right)$ and $t=\psi(\theta)=\psi\left(\varphi_{3}\right)^{-1} x a_{1}^{2} a_{2}\left(t_{1}\right)$. We have seen that $\psi\left(\varphi_{3}\right)=f_{3}$ and so, using Corollary 1.4, we can see that $t=e a_{1} a_{2} e$ where $\varepsilon$ is the curve shown in Figure 16. 
Recall that by relation (2) (see the proof of Lemma 1.2), one has $a_{1} t_{1}=t_{1} a_{2}$ and $a_{2} t_{1}=t_{1} a_{1}$. Then one can compute:

$$
\begin{aligned}
& s m_{5} s m_{4} s m_{3} s m_{2} s m_{1} s \\
& =\left(a_{1} b_{1} a_{1}\right)\left(\overline{a_{1}} d_{2,3}\right)\left(a_{1} b_{1} a_{1}\right)\left(d_{2,3} \overline{a_{1}} \bar{t}\right)\left(a_{1} b_{1} a_{1}\right)\left(\overline{a_{1}} d_{2,3}\right) \\
& \times\left(a_{1} b_{1} a_{1}\right)\left(\overline{d_{2,3}} t\right)\left(a_{1} b_{1} a_{1}\right)\left(\overline{a_{1}} d_{2,3}\right)\left(a_{1} b_{1} a_{1}\right) \\
& =a_{1} b_{1} a_{1} d_{2,3} b_{1} d_{2,3} \bar{t} a_{1} b_{1} d_{2,3} a_{1} b_{1} a_{1} \overline{d_{2,3}} t a_{1} b_{1} d_{2,3} a_{1} b_{1} a_{1} \text { by }(0) \\
& =\left(a_{1} b_{1} d_{2,3}\right)^{2} \bar{t} b_{1} a_{1} b_{1} d_{2,3} b_{1} \overline{d_{2,3}} a_{1} t a_{1} b_{1} d_{2,3} a_{1} b_{1} a_{1} \text { by (0) and (1) } \\
& =\left(a_{1} b_{1} d_{2,3}\right)^{2} \bar{t} b_{1} a_{1} d_{2,3} b_{1} d_{2,3} \overline{d_{2,3}} a_{2} t a_{2} b_{1} d_{2,3} a_{1} b_{1} a_{1} \text { by (1) and } \\
& =\left(a_{1} b_{1} d_{2,3}\right)^{2} \bar{t} a_{2} b_{1} d_{2,3} a_{1} b_{1} \text { e } a_{1} a_{2} e b_{1} d_{2,3} a_{1} b_{1} a_{1} a_{2} \text { by (0) } \\
& =\left(a_{1} b_{1} d_{2,3}\right)^{2} a_{1} \bar{t} b_{1} d_{2,3} a_{1} e b_{1} a_{1} b_{1} a_{2} e d_{2,3} a_{1} b_{1} a_{1} a_{2} \text { by (0) and (2) } \\
& =\left(a_{1} b_{1} d_{2,3}\right)^{2} a_{1} \bar{t} b_{1} d_{2,3} a_{1} e a_{1} b_{1} a_{1} a_{2} e d_{2,3} a_{1} b_{1} a_{1} a_{2} \text { by (1) } \\
& =\left(a_{1} b_{1} d_{2,3}\right)^{2} a_{1} \bar{t} b_{1} d_{2,3} e a_{1} e b_{1} a_{1} a_{2} e d_{2,3} a_{1} b_{1} a_{1} a_{2} \text { by (1) } \\
& =\left(a_{1} b_{1} d_{2,3}\right)^{2} a_{1} \bar{e} \overline{a_{1}} \overline{a_{2}} \bar{e} b_{1} d_{2,3} e a_{1} b_{1} t d_{2,3} a_{1} b_{1} a_{1} a_{2} \text { by (0) } \\
& =\left(a_{1} b_{1} d_{2,3}\right)^{2} a_{1} \bar{e} \overline{a_{1}} \overline{a_{2}} b_{1} d_{2,3} a_{1} b_{1} t d_{2,3} a_{1} b_{1} a_{1} a_{2} \text { by }(0) \\
& =\left(a_{1} b_{1} d_{2,3}\right)^{2} a_{1} \bar{e} \overline{a_{1}} b_{1} a_{1} d_{2,3} b_{1} \overline{a_{2}} t d_{2,3} a_{1} b_{1} a_{1} a_{2} \text { by }(0) \\
& =\left(a_{1} b_{1} d_{2,3}\right)^{2} a_{1} \bar{e} b_{1} a_{1} \overline{b_{1}} d_{2,3} b_{1} t \overline{a_{1}} d_{2,3} a_{1} b_{1} a_{1} a_{2} \text { by (1) and (2) } \\
& =\left(a_{1} b_{1} d_{2,3}\right)^{2} a_{1} b_{1} \bar{e} a_{1} d_{2,3} b_{1} \overline{d_{2,3}} t d_{2,3} b_{1} a_{1} a_{2} \text { by (0) and (1) } \\
& =\left(a_{1} b_{1} d_{2,3}\right)^{2} a_{1} b_{1} d_{2,3} \bar{e} a_{1} b_{1} t b_{1} a_{1} a_{2} \text { by }(0) \\
& =\left(a_{1} b_{1} d_{2,3}\right)^{3} \bar{e} a_{1} b_{1} e a_{1} a_{2} e b_{1} a_{1} a_{2} \\
& =\left(a_{1} b_{1} d_{2,3}\right)^{3} \bar{e} a_{1} e b_{1} a_{1} b_{1} a_{2} e a_{1} a_{2} \text { by }(0) \\
& =\left(a_{1} b_{1} d_{2,3}\right)^{3} a_{1} e \overline{a_{1}} a_{1} b_{1} a_{1} a_{2} e a_{1} a_{2} \text { by (1) } \\
& =\left(a_{1} b_{1} d_{2,3}\right)^{3} a_{1} b_{1} \text { e } a_{1} a_{2} e a_{1} a_{2} \text { by (0) } \\
& =\left(a_{1} b_{1} d_{2,3}\right)^{4} \overline{d_{2,3}} t a_{1} a_{2} \\
& =a_{1}^{2}\left(x d_{2,3}\right)^{2} \overline{d_{2,3}} t a_{1} a_{2} \text { by Lemma 1.2. }
\end{aligned}
$$

It is known that $a_{i}, x$ and $d_{2,3}$ are elements of $H^{\prime}$. Since $\psi\left(\varphi_{3}\right)=a_{1}^{2}\left(x d_{2,3}\right)^{2} \overline{a_{k_{i}}} \in$ $H^{\prime}$, one has $t=\psi(\theta)=\psi\left(\varphi_{3}\right) x a_{1}^{2} a_{2}\left(t_{1}\right) \in H^{\prime}$. This concludes the proof of (viii).

This ends the proof that $\psi: \mathcal{M} \rightarrow G$ is a homomorphism. One has $\varphi \psi=$ $I d_{\mathcal{M}}$. So, to prove Theorem A, it is enough to show that $\psi$ is onto. Let $\alpha$ be a s.c.c. in $\Sigma$. We have to show that $a$ is in $K$, the subgroup of $G$ generated by $\left\{a_{i}{ }^{\prime} \mathrm{s}, d_{i j}{ }^{\prime} \mathrm{s}, x, t_{i}{ }^{\prime} \mathrm{s}, s\right\}$ (one has $\psi(\mathcal{M})=K$ ).

First case: $\alpha$ does not separate $\Sigma$. There exists a diffeomorphism $h$ of $\Sigma$ such that $h\left(\alpha_{1}\right)=\alpha$. If we decompose $h$ into a product of elements of $\left\{\alpha_{i}{ }^{\prime} \mathrm{s}, \delta_{i j}{ }^{\prime} \mathrm{s}, \xi, \theta_{i}{ }^{\prime} \mathrm{s}\right.$, $\sigma\}$ (these elements generate $\mathcal{M}$ by Corollary 2.2) and set $m=\psi(h)$, then Corollary 1.4 implies $a=m\left(a_{1}\right) \in K$.

Second case: $\alpha$ separates in $\Sigma$. Denote by $g_{\alpha}$ the genus of the component of $\Sigma \backslash \alpha$ which does not contain $\delta$, and by $r_{\alpha}$ its number of boundary components. Up to diffeomorphism, $\alpha$ is the curve shown in Figure 19.

If, in $\Sigma \backslash\left(\bigcup \alpha_{i}\right), \alpha$ encircles only one hole, then $\alpha=\alpha_{g+1}$ and $a \in K$. If $\alpha$ encircles two holes, then $\alpha$ is one of the curves $\delta_{2 g-1,2 g}$ or $\delta_{2 g+1,2 g+2}$ and $a \in$ 


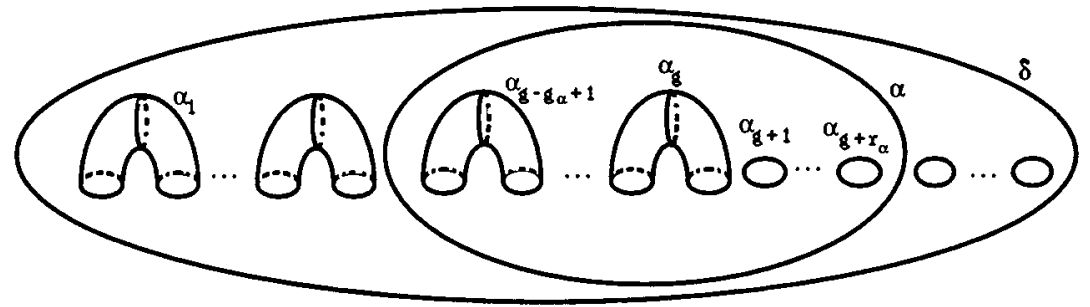

Figure 19

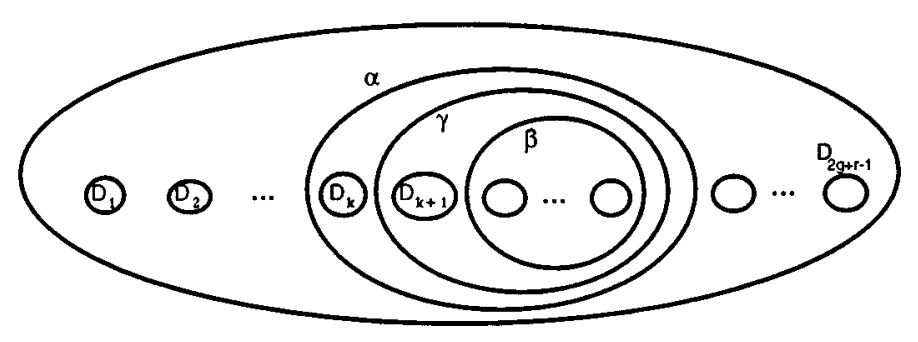

FiguRe 20

$K$. Otherwise, one can find three curves $\beta, \gamma, \lambda$ verifying the lantern relation $a a_{i_{k}} a_{i_{k+1}} b=d_{k, k+1} c l$ in $G$ and such that if one of them separates, it encircles fewer holes than $\alpha$ does (see Figure 20). Then we conclude, using the first case and an inductive argument.

We have proved the first part of Theorem A when $r \geq 1$. If $r=0$, it is clear that the presentation given by Wajnryb in [W] together with Theorem 1.3 implies Theorem A. The last statement of the theorem was proven in Theorem 1.6.

2.2. Proof of Theorem B. Harer proved in $[\mathrm{H}]$ that, when $g \geq 3, \mathcal{M}_{g, r}$ is generated by twists along non-separating curves. The following two lemmas tell us what happens in genus one and two.

Lemma 2.5. For any $r \geq 0$, the twists on non-separating curves generate $\mathcal{M}_{2, r}$.

Proof. We only need to prove that a twist on a separating curve can be decomposed into a product of twists on non-separating curves. So, let $\alpha$ be a separating curve in $\Sigma_{2, r}$.

First case. One of the two components of $\Sigma \backslash \alpha$ has genus zero. Then the star $\left(\alpha_{1}, \alpha_{2}, \alpha_{3}, \beta\right)$ (see Figure 21) gives $\left(\tau_{\alpha_{1}} \tau_{\alpha_{2}} \tau_{\alpha_{3}} \tau_{\beta}\right)^{3}=\tau_{\alpha} \tau_{\delta_{1}} \tau_{\delta_{2}}$.

Second case. The two components of $\Sigma \backslash \alpha$ have genus one. The chain $\left(\alpha_{1}, \beta, \alpha_{2}\right)$ (see Figure 22) gives $\left(\tau_{\alpha_{1}} \tau_{\beta} \tau_{\alpha_{2}}\right)^{4}=\tau_{\alpha} \tau_{\delta}$ and we conclude using the first case.

Lemma 2.6. For any $r \geq 1, \mathcal{M}_{1, r}$ is generated by twists on $r-1$ boundary curves together with those on non-separating curves.

This lemma will be proved in Section 5 .

We shall now prove Theorem B. From now on, $\Sigma$ will denote a surface of genus $g \geq 2$ with $r$ boundary components $(r \geq 0)$. Let $G^{\prime}$ be a group with generators 


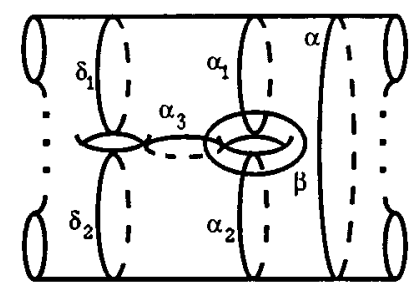

FIGURE 21

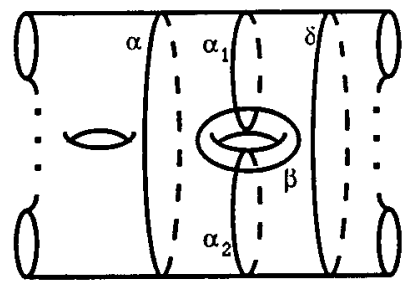

FiguRe 22

$\left\{a \equiv a_{\alpha} / \alpha\right.$ non-separating s.c.c. in $\left.\Sigma\right\}$ and relations (I)', (II)' and (III)'. Since $G^{\prime}$ is a quotient of $S$, the relations which have been shown in Section 1 will be true in $G^{\prime}$. In particular, all lanterns, stars and chains are equal to one if the curves involved do not separate (Theorem 1.6).

Define $\varphi: G^{\prime} \rightarrow \mathcal{M}$ by $\varphi(a)=\alpha . \varphi$ is clearly a homomorphism. Harer's result and Lemma 2.5 show that $\varphi$ is onto. We shall construct an inverse morphism $\psi: \mathcal{M} \rightarrow G^{\prime}$. To do this, we consider $\mathcal{M}$ given by the presentation of Theorem A. So, let $\alpha$ be a s.c.c. in $\Sigma$. If $\alpha$ does not separate, we set $\psi(\alpha)=a$. Otherwise, there are the two following cases.

First case. One of the two components of $\Sigma \backslash \alpha$ has genus greater than or equal to two. Consider the curves shown in Figure 21 and define $\psi(\alpha)$ by $\psi(\alpha)=$ $\left(a_{1} a_{2} a_{3} b\right)^{3} \overline{d_{1}} \overline{d_{2}}$. We have to show that $\psi(\alpha)$ is independent of the choice of the star. So, let $\left(\alpha_{1}^{\prime}, \alpha_{2}^{\prime}, \alpha_{3}^{\prime}, \beta^{\prime}\right)$ be a second star and denote $\left(a_{1} a_{2} a_{3} b\right)^{3} \overline{d_{1}} \overline{d_{2}}$ by $a$ and $\left(a_{1}^{\prime} a_{2}^{\prime} a_{3}^{\prime} b^{\prime}\right)^{3} \overline{d_{1}^{\prime}} \overline{d_{2}^{\prime}}$ by $a^{\prime}$. We want to show that $a=a^{\prime}$.

If all the curves involved lie in the same component $\Sigma_{1}$ of $\Sigma \backslash \alpha$, there is a diffeomorphism $h$ of $\Sigma_{1}$ which sends the first star onto the second one fixing $\alpha$ (this is a consequence of the classification of surfaces). Decompose $h$ into a product of twists on non-separating curves in $\Sigma_{1}$ (it can be done since $\Sigma_{1}$ has genus greater than or equal to two) and extend it to $\Sigma$ by the identity. Then, if $m$ denotes the word of $G^{\prime}$ associated to $h$, one has $a^{\prime}=m(a)$ using Corollary 1.4. But if $\gamma$ is a curve in the decomposition of $h$, then $\gamma$ and $\alpha$ are disjoint. So, one has

$$
\gamma=\tau_{\alpha}(\gamma)=\left(\tau_{\alpha_{1}} \tau_{\alpha_{2}} \tau_{\alpha_{3}} \tau_{\beta}\right)^{4} \tau_{\delta_{1}}^{-1} \tau_{\delta_{2}}^{-2}(\gamma)
$$

and by Corollary 1.4, $a(c)=a$. This proves that $m$ commutes with $a$ and so $a^{\prime}=a$.

We suppose now that the two stars lie in distinct components of $\Sigma \backslash \alpha$ (see Figure 23). 

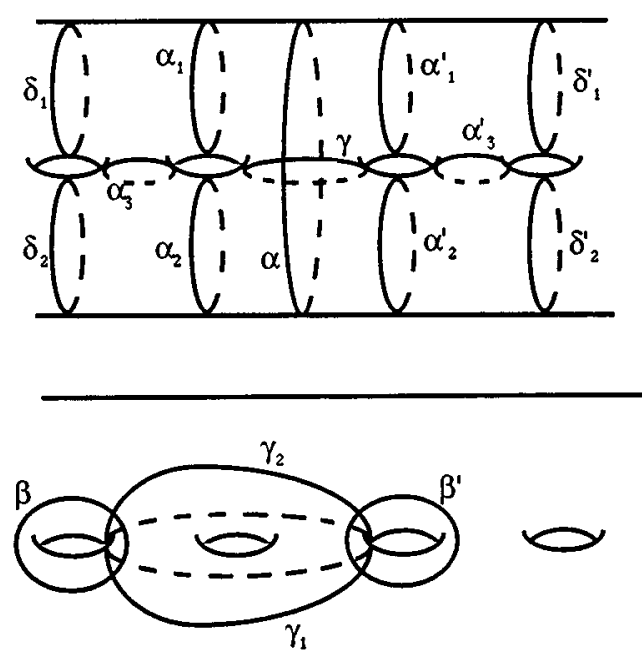

FIGURE 23

By Lemma 1.7 and stars and chains relations $E_{0}$ and $C_{0}$, one has

$$
\begin{gathered}
\left(c b a_{1} a_{2} b c\right)\left(a_{3} b a_{1} a_{2} b a_{3}\right)=a_{1}^{\prime} a_{2}^{\prime} d_{1} d_{2}, \\
\left(c b^{\prime} a_{1}^{\prime} a_{2}^{\prime} b^{\prime} c\right)\left(a_{3}^{\prime} b^{\prime} a_{1}^{\prime} a_{2}^{\prime} b^{\prime} a_{3}^{\prime}\right)=a_{1} a_{2} d_{1}^{\prime} d_{2}^{\prime} .
\end{gathered}
$$

Thus, we obtain, using Lemma 1.2 and (0),

$$
\begin{aligned}
a & =\left(a_{1} a_{2} a_{3} b\right)^{3} \overline{d_{1}} \overline{d_{2}} \\
& =\left[\left(a_{1} a_{2}\right)\left(b a_{1} a_{2} b\right)\left(a_{3} b a_{1} a_{2} b a_{3}\right)\right]\left[\left(a_{1}^{\prime} a_{2}^{\prime}\right)\left(\overline{a_{3}} \bar{b} \overline{a_{1}} \overline{a_{2}} \bar{b} \overline{a_{3}}\right)\left(\bar{c} \bar{b} \overline{a_{1}} \overline{a_{2}} \bar{b} \bar{c}\right)\right] \\
& =a_{1} a_{2} a_{1}^{\prime} a_{2}^{\prime}\left[b a_{1} a_{2} b \bar{c} \bar{b} \overline{a_{1}} \overline{a_{2}} \bar{b}\right] \bar{c}
\end{aligned}
$$

and, similarly,

$$
a^{\prime}=\left(a_{1}^{\prime} a_{2}^{\prime} a_{3}^{\prime} b^{\prime}\right)^{3} \overline{d_{1}^{\prime}} \overline{d_{2}^{\prime}}=a_{1}^{\prime} a_{2}^{\prime} a_{1} a_{2}\left[b^{\prime} a_{1}^{\prime} a_{2}^{\prime} b^{\prime} \bar{c} \overline{b^{\prime}} \overline{a_{1}^{\prime}} \overline{a_{2}^{\prime}} \overline{b^{\prime}}\right] \bar{c} .
$$

But one can check that $\tau_{\beta} \tau_{\alpha_{1}} \tau_{\alpha_{2}} \tau_{\beta}(\gamma)=\tau_{\beta^{\prime}} \tau_{\alpha_{1}^{\prime}} \tau_{\alpha_{2}^{\prime}} \tau_{\beta^{\prime}}(\gamma)$. So, if $\gamma^{\prime}$ denotes this curve, one has (by Corollary 1.4)

$$
a=a_{1} a_{2} a_{1}^{\prime} a_{2}^{\prime} \overline{c^{\prime}} \bar{c}=a^{\prime} .
$$

Lemma 2.7. Let $\delta$ be a separating curve in $\Sigma$ such that $\Sigma \backslash \delta$ has a component of genus greater than two. Let $h$ be a diffeomorphism of $\Sigma$ which is decomposed into a product of twists along non-separating curves. Then, if $\delta^{\prime}=h(\delta)$ and $m$ is the word associated to $h$ in $G^{\prime}$, one has $\psi\left(\delta^{\prime}\right)=m \psi(\delta) \bar{m}$.

Proof. Let $E=\left(\alpha_{1}, \alpha_{2}, \alpha_{3}, \beta\right)$ be a star such that $\psi(\delta)=\left(a_{1} a_{2} a_{3} b\right)^{3} \overline{d_{1}} \overline{d_{2}}$ (Figure 21). Then, if $E^{\prime}=\left(\alpha_{1}^{\prime}, \alpha_{2}^{\prime}, \alpha_{3}^{\prime}, \beta^{\prime}\right)$ is the image of $E$ by $h$, one has $\psi\left(\delta^{\prime}\right)=$ $\left(a_{1}^{\prime} a_{2}^{\prime} a_{3}^{\prime} b^{\prime}\right)^{3} \overline{d_{1}^{\prime}} \overline{d_{2}^{\prime}}$. Furthermore, Corollary 1.4 gives $a_{k}^{\prime}=m\left(a_{k}\right), \quad b^{\prime}=m(b)$ and $d_{k}^{\prime}=m\left(d_{k}\right)$, which imply the required result.

Second case. The two components of $\Sigma \backslash \alpha$ have genus one. Consider the curves shown in Figure 22 and define $\psi(\alpha)$ by $\psi(\alpha)=\left(a_{1} b a_{2}\right)^{4} \overline{\psi(\delta)}$ (according to the first case, $\psi(\delta)$ is well defined). We have to show that $\psi(\alpha)$ is independent of the choice 


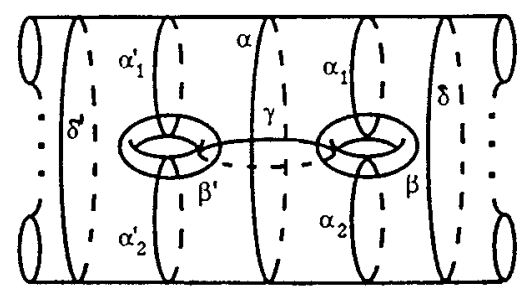

Figure 24

of the chain. So, let $\left(\alpha_{1}^{\prime}, \beta^{\prime}, \alpha_{2}^{\prime}\right)$ be a second chain and denote $\left(a_{1} b a_{2}\right)^{4} \overline{\psi(\delta)}$ by $a$ and $\left(a_{1}^{\prime} b^{\prime} a_{2}^{\prime}\right)^{4} \overline{\psi\left(\delta^{\prime}\right)}$ by $a^{\prime}$.

If the two chains lie in the same component $\Sigma_{1}$ of $\Sigma \backslash \alpha$, there is a diffeomorphism $h$ of $\Sigma_{1}$ which sends one of the two chains to the other one fixing $\alpha$. By Lemma 2.6, one can decompose $h$ into a product of twists along non-separating curves or boundary curves. But since a twist on a boundary curve is central, one can suppose that $h$ is a product of twists along only non-separating curves. Extend $h$ to $\Sigma$ by identity. Then, if $m$ is the word in $G^{\prime}$ corresponding to the decomposition of $h$, one has by Corollary 1.4 and Lemma $2.7 a^{\prime}=m(a)$. At last, since all the curves involved in the decomposition of $m$ are disjoint from $\alpha, m$ commutes with $a$ by Corollary 1.4.

We now suppose that the chains lie in distinct components of $\Sigma \backslash \alpha$ (Figure 24). We set

$$
\begin{gathered}
X_{1}=a_{1} a_{2}, \quad X_{2}=b X_{1} b, \quad X_{3}=c X_{2} c, \quad X_{1}^{\prime}=a_{1}^{\prime} a_{2}^{\prime}, \\
X_{2}^{\prime}=b^{\prime} X_{1}^{\prime} b^{\prime}, \text { and } X_{3}^{\prime}=c X_{2}^{\prime} c .
\end{gathered}
$$

Then, one has, using Lemma 1.2, $a=X_{1}^{2} X_{2}^{2} \overline{\psi(\delta)}$ with $\psi(\delta)=X_{1} X_{2} X_{3} \overline{a_{1}^{\prime}} \overline{a_{2}^{\prime}}$ and $a^{\prime}=X_{1}^{\prime 2} X_{2}^{\prime 2} \overline{\psi\left(\delta^{\prime}\right)}$ with $\psi\left(\delta^{\prime}\right)=X_{1}^{\prime} X_{2}^{\prime} X_{3}^{\prime} \overline{a_{1}} \frac{2}{a_{2}}$. Thus,

$$
a=\left(X_{1} X_{2}\right)^{2} \overline{X_{1}} \overline{X_{2}} \overline{X_{3}} X_{1}^{\prime}=X_{1} X_{2} \bar{c} \overline{X_{2}} \bar{c} X_{1}^{\prime} \quad \text { and } \quad a^{\prime}=X_{1}^{\prime} X_{2}^{\prime} \bar{c} \overline{X_{2}^{\prime}} \bar{c} X_{1} .
$$

But since $\tau_{\beta} \tau_{\alpha_{1}} \tau_{\alpha_{2}} \tau_{\beta}(\gamma)=\tau_{\beta^{\prime}} \tau_{\alpha_{1}^{\prime}} \tau_{\alpha_{2}^{\prime}} \tau_{\beta^{\prime}}(\gamma)$, by Corollary 1.4 we have $X_{2}(c)=$ $X_{2}^{\prime}(c)$. Thus, we obtain $a=X_{1} X_{2}^{\prime} \bar{c} \overline{X_{2}^{\prime}} \bar{c} X_{1}^{\prime}=a^{\prime}$.

We have proved that $\psi: \mathcal{M} \rightarrow G^{\prime}$ is well defined. It remains to prove that $\psi$ is a homomorphism. To do this, it is enough to show that the relations (I) and (IV) are mapped by $\psi$ into true relations in $G^{\prime}$.

The braids. Let $\lambda$ and $\mu$ be two curves in $\Sigma, \nu=\tau_{\mu}(\lambda)$. We have to prove that $\psi(\nu)=\psi(\mu) \psi(\lambda) \psi(\mu)^{-1}$. This is shown by Corollary 1.4 if $\lambda$ does not separate. Otherwise, if a component of $\Sigma \backslash \lambda$ has genus greater than two, the relation is still true by Lemma 2.7 .

Finally, let us suppose that $\lambda$ separates and that the two components of $\Sigma \backslash \lambda$ have genus one. Let $C\left(\alpha_{1}, \beta, \alpha_{2}\right)$ be a chain such that $\psi(\lambda)=\left(a_{1} b a_{2}\right)^{4} \overline{\psi(\delta)}$ (see Figure 22) and denote by $\alpha_{1}^{\prime}, \beta^{\prime}, \alpha_{2}^{\prime}$ and $\delta^{\prime}$ the respective image of $\alpha_{1}, \beta, \alpha_{2}$ and $\delta$ under $\tau_{\mu}$. Then, one has $\psi(\nu)=\left(a_{1}^{\prime} b^{\prime} a_{2}^{\prime}\right)^{4} \overline{\psi\left(\delta^{\prime}\right)}$.

But there are some non-separating curves $\varepsilon_{1}, \ldots, \varepsilon_{p}$ such that $\psi(\mu)$ is equal to the product $e_{1} \ldots e_{p}$. Thus, by Corollary 1.4, one has $a_{k}^{\prime}=\psi(\mu)\left(a_{k}\right), b^{\prime}=\psi(\mu)(b)$ and, by Lemma $2.7, \psi\left(\delta^{\prime}\right)=\psi(\mu)(\psi(\delta))$. So, we obtain $\psi(\nu)=\psi(\mu) \psi(\lambda) \psi(\mu)^{-1}$. 


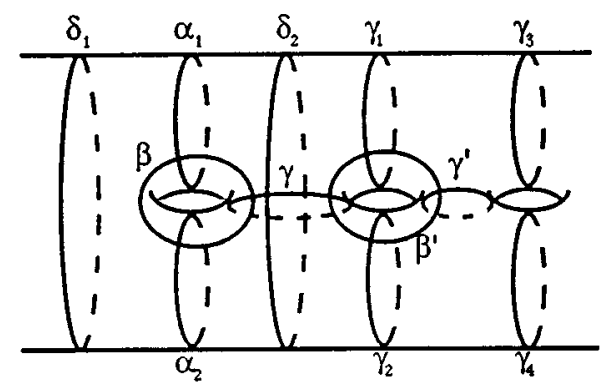

FiguRE 25

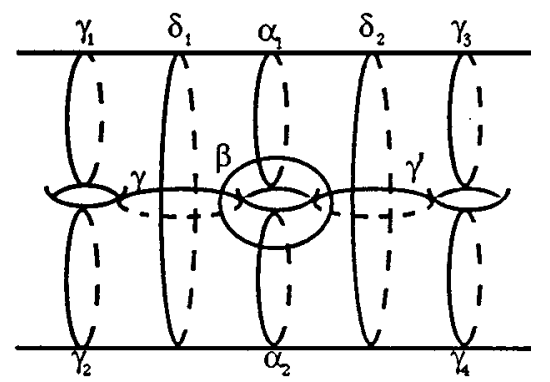

Figure 26

Remark. Since the braids relations are mapped by $\psi$ into true relations, one has in $G^{\prime}$, for any disjoint s.c.c. $\alpha$ and $\beta$ in $\Sigma$, the relation

$$
\psi(\alpha) \psi(\beta)=\psi(\beta) \psi(\alpha) .
$$

Lemma 2.8. The chain relations are satisfied in $G^{\prime}$.

Proof. Let $\left(\alpha_{1}, \beta, \alpha_{2}\right)$ be a chain $C$, and $\delta_{1}$ and $\delta_{2}$ its boundary components. If $\delta_{1}$ and $\delta_{2}$ do not separate, the relation is satisfied by (II)' and Theorem 1.6. So, we suppose that $\delta_{1}$ and $\delta_{2}$ separate in $\Sigma$. If $g$ is equal to two, the relation is satisfied by definition of $\psi\left(\delta_{k}\right)$. And when $g \geq 3$, two different cases occur, which are described by Figures 25 and 26 (we have to consider the second case only if $g=3$ ).

First case (Figure 25). The definition of $\psi\left(\delta_{k}\right)$ gives $\psi\left(\delta_{1}\right)=X_{1} X_{2} X_{3} \overline{Y_{1}}$ and $\psi\left(\delta_{2}\right)=Y_{1} Y_{2} Y_{3}^{\prime} \overline{c_{3}} \overline{c_{4}}$, where

$$
\begin{aligned}
& X_{1}=a_{1} a_{2}, \quad X_{2}=b X_{1} b, \quad X_{3}=c X_{2} c, \\
& Y_{1}=c_{1} c_{2}, \quad Y_{2}=b^{\prime} Y_{1} b^{\prime}, \quad Y_{3}^{\prime}=c^{\prime} Y_{2} c^{\prime} .
\end{aligned}
$$

Furthermore, if $Y_{3}=c Y_{2} c$, one has by Lemma $1.7 Y_{3} Y_{3}^{\prime}=a_{1} a_{2} c_{3} c_{4}$. Thus, we obtain

$$
\begin{aligned}
\psi\left(\delta_{1}\right) \psi\left(\delta_{2}\right) & =X_{1} X_{2} X_{3} Y_{2} Y_{3}^{\prime} \overline{c_{3}} \overline{c_{4}}=\left(X_{1} X_{2} X_{3} Y_{2}\right)\left(\overline{Y_{3}} X_{1}\right) \\
& =X_{1}^{2} X_{2}^{2} \overline{X_{2}} c X_{2} c Y_{2} \bar{c} \overline{Y_{2}} \bar{c}
\end{aligned}
$$

At last, since $\overline{X_{2}}(c)=c Y_{2}(c)$ (Corollary 1.4), we have $X_{1}^{2} X_{2}^{2}=\psi\left(\delta_{1}\right) \psi\left(\delta_{2}\right)$. 

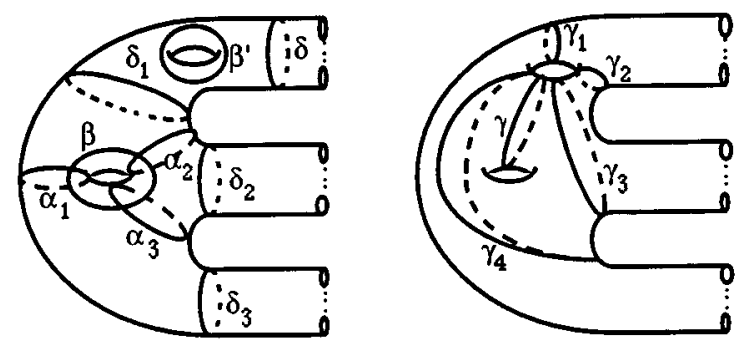

FiguRE 27

Second case (Figure 26). Set $X_{1}=a_{1} a_{2}, X_{2}=b X_{1} b, X_{3}=c X_{2} c$ and $X_{3}^{\prime}=$ $c^{\prime} X_{2} c^{\prime}$. Then, by definition, we have

$$
\psi\left(\delta_{1}\right)=X_{1} X_{2} X_{3}^{\prime} \overline{c_{3}} \overline{c_{4}} \quad \text { and } \quad \psi\left(\delta_{2}\right)=X_{1} X_{2} X_{3} \overline{c_{1}} \overline{c_{2}}
$$

and so

$$
\psi\left(\delta_{1}\right) \psi\left(\delta_{2}\right)=X_{1}^{2} X_{2}^{2} X_{3}^{\prime} X_{3} \overline{c_{1}} \overline{c_{2}} \overline{c_{3}} \overline{c_{4}} .
$$

Then, using the equality $X_{3}^{\prime} X_{3}=c_{1} c_{2} c_{3} c_{4}$ (given by Lemma 1.7), one obtains the required result.

The stars. Let $\left(\alpha_{1}, \alpha_{2}, \alpha_{3}, \beta\right)$ be a star $E$ in $\Sigma$, and let $\delta_{1}, \delta_{2}$, and $\delta_{3}$ be the boundary components. If all these curves do not separate $\Sigma, E$ is satisfied in $G^{\prime}$ by (II)', (III)' and Theorem 1.6. If only one curve $\delta_{i}$ separates, $E$ is satisfied by definition of $\psi\left(\delta_{i}\right)$. So, let us suppose that $\delta_{1}, \delta_{2}$ and $\delta_{3}$ separate $\Sigma$. Since $g \geq 2$, one can suppose that the component of $\Sigma \backslash \delta_{1}$ which does not contain $\delta_{2}$ is of genus greater than or equal to one. Consider the curves shown on Figure 27, and set

$$
\begin{gathered}
X_{1}=a_{3} c, \quad X_{2}=b X_{1} b, \quad X_{3}=a_{1} X_{2} a_{1}, \quad X_{3}^{\prime}=a_{2} X_{2} a_{2}, \\
Y_{1}=c_{1} c_{2}, \quad Y_{2}=b^{\prime} Y_{1} b^{\prime}, \quad Y_{3}=c Y_{2} c .
\end{gathered}
$$

By definition of $\psi\left(\delta_{2}\right), \psi\left(\delta_{3}\right)$ and $\psi(\delta)$, one has

$$
X_{1} X_{2} X_{3}=c_{1} c_{3} \psi\left(\delta_{3}\right), X_{1} X_{2} X_{3}^{\prime}=c_{2} c_{4} \psi\left(\delta_{2}\right), Y_{1} Y_{2} Y_{3}=a_{1} a_{2} \psi(\delta)
$$

and Lemma 2.8 gives $X_{1}^{2} X_{2}^{2}=c_{3} c_{4}$ and $Y_{1}^{2} Y_{2}^{2}=\psi\left(\delta_{1}\right) \psi(\delta)$. From these relations we obtain

$$
\begin{aligned}
\psi\left(\delta_{2}\right) \psi\left(\delta_{3}\right) & =X_{1} X_{2} X_{3}^{\prime} \overline{c_{2}} \overline{c_{4}} X_{1} X_{2} X_{3} \overline{c_{1}} \overline{c_{3}} \\
& =X_{1}^{2} X_{2}^{2} \overline{c_{1}} \overline{c_{2}} \overline{c_{3}} \overline{c_{4}} X_{3} X_{3}^{\prime} \quad \text { by }(\mathcal{B}) \text { and Lemma } 1.2 \\
& =\overline{c_{1}} \overline{c_{2}}\left(a_{1} b a_{3} c b a_{1}\right)\left(a_{2} b a_{3} c b a_{2}\right) \\
& =\overline{c_{1}} \overline{c_{2}}\left(c b a_{1} a_{2} b c\right)\left(a_{3} b a_{1} a_{2} b a_{3}\right) \quad \text { by }(\mathcal{B}), \\
\psi\left(\delta_{1}\right) \psi\left(\delta_{2}\right) \psi\left(\delta_{3}\right) & =\left(Y_{1}^{2} Y_{2}^{2} \psi(\delta)^{-1}\right) \overline{c_{1}} \overline{c_{2}}\left(c b a_{1} a_{2} b c\right)\left(a_{3} b a_{1} a_{2} b a_{3}\right) \\
& =Y_{1}^{2} Y_{2}^{2}\left(\overline{Y_{1}} \overline{Y_{2}} \overline{Y_{3}} a_{1} a_{2}\right) \overline{Y_{1}}\left(c b a_{1} a_{2} b c\right)\left(a_{3} b a_{1} a_{2} b a_{3}\right) \\
& =a_{1} a_{2} Y_{2} \bar{c} \overline{Y_{2}} \bar{c}\left(c b a_{1} a_{2} b c\right)\left(a_{3} b a_{1} a_{2} b a_{3}\right) \quad \text { by }(0) .
\end{aligned}
$$

Now, set $Z_{1}=a_{1} a_{2}, Z_{2}=b Z_{1} b$ and $Z_{3}=a_{3} Z_{2} a_{3}$. Then, using Corollary 1.4 one can check that $Y_{2}(c)=Z_{2}(c)$. Thus, we obtain

$$
\psi\left(\delta_{1}\right) \psi\left(\delta_{2}\right) \psi\left(\delta_{3}\right)=Z_{1}\left[Z_{2} \bar{c} \overline{Z_{2}} \bar{c}\right]\left[c Z_{2} c\right] Z_{3}=Z_{1} Z_{2} Z_{3} .
$$


Lemma 1.2 shows that this last relation is the star $E$.

This proves that $\psi$ is a homomorphism. One has clearly $\psi \circ \varphi=\operatorname{Id}_{G^{\prime}}$ so $\varphi$ is a monomorphism from $G^{\prime}$ to $\mathcal{M}$. Since $\varphi$ is onto, this concludes the proof of Theorem B.

\section{Mapping Class Group With $p_{1}$-STRUCture}

A Topological Quantum Field Theory is a functor from a cobordism category to a category of modules with suitable properties (see for instance [A1]). When we consider the cobordism category of 2-dimensional surfaces and 3-dimensional cobordisms, this functor produces projective representations of mapping class groups. One can linearize this representation by considering a certain central extension (G. Masbaum and J. Roberts describe some of these extensions in $[\mathrm{M}-\mathrm{R}]$ ).

In [BHMV2], the authors construct a TQFT $V_{p}$ from the Kauffman bracket. In order to solve the framing anomaly, all the manifolds are equipped with $p_{1^{-}}$ structure. The central extension obtained this way is the mapping class group with $p_{1}$-structure and the goal of this section is to give a presentation of this group.

\subsection{Definitions.}

Definition 3.1. Let $X$ be the homotopy fiber of the map $p_{1}: B O \rightarrow K(\mathbb{Z}, 4)$ corresponding to the first Pontryagin class of the universal stable bundle $\gamma$ over $B O$. A $p_{1}$-structure on a manifold $M$ is a lifting of a classifying map of the stable tangent bundle of $M$ to $X$.

Definition 3.2. A homotopy between two $p_{1}$-structures $\Phi_{0}$ and $\Phi_{1}$ on a manifold $M$ is a $p_{1}$-structure $\Phi$ on $M \times I$ such that $\Phi_{\mid M \times 0}$ and $\Phi_{\mid M \times 1}$ coincide respectively with $\Phi_{0}$ and $\Phi_{1}$.

If $N$ is a submanifold of $M$, one can define a homotopy rel $N$ in the obvious way.

Remarks. (i) A $p_{1}$-structure up to homotopy is the analogue of a spin structure, where the second Stiefel-Whitney class $w_{2}$ is replaced by the first Pontryagin class $p_{1}$.

(ii) We consider actual $p_{1}$-structure rather than the homotopy class of such a structure. This allows manifolds with $p_{1}$-structure to be glued along parts of their boundary.

For the relevant classical algebraic topology or structure on manifolds, see for instance $[\mathrm{S}]$ or $[\mathrm{Sw}]$. The following fact is easily proven using obstruction theory.

Proposition 3.3. If $M$ is a manifold and $N$ a submanifold of $M$, then, the set of homotopy class rel $N$ of $p_{1}$-structure on $M$ is affinely isomorphic to $H^{3}(M, N ; \mathbb{Z})$.

Thus, manifolds of dimension less than or equal to two admit a unique $p_{1}$ structure up to homotopy, and the set of homotopy class rel boundary of $p_{1^{-}}$ structure on an oriented, compact, connected 3-manifold is affinely isomorphic to $\mathbb{Z}$.

Now, let $\Sigma$ be an oriented, connected surface and $\varphi$ a given $p_{1}$-structure on $\Sigma$. We shall define $\widetilde{\mathcal{M}}_{\Sigma}$, the mapping class group with $p_{1}$-structure. For $f \in \mathcal{M}_{\Sigma}$, we provide $\partial(\Sigma \times I)$ with the following $p_{1}$-structure: $\varphi \circ f$ on $\Sigma \times 0$ and $\varphi$ on $(\Sigma \times 1 \cup \partial \Sigma \times I)$. This can be extended to $\Sigma \times I$, and $P_{f}$, the set of homotopy class of such extensions, is affinely isomorphic to $\mathbb{Z}$. 
Definition 3.4. The mapping class group with $p_{1}$-structure is the set of all pairs $(f, F)$ where $f \in \mathcal{M}_{\Sigma}$ and $F \in P_{f}$, together with the obvious composition.

Remark. Atiyah ([A2]) has previously defined this group in a different way.

The forgetful map $\mu$ is an epimorphism from $\tilde{\mathcal{M}}_{\Sigma}$ to $\mathcal{M}_{\Sigma}$, with kernel $\mathbb{Z}$. Let us denote by $u$ a generator of $\operatorname{Ker} \mu$. Since to modify a given $p_{1}$-structure on $\Sigma \times I$, it is enough to change it in a small 3 -ball, $u$ is in the center of $\widetilde{\mathcal{M}}_{\Sigma}$. So, we obtain the following central extension of $\mathcal{M}_{\Sigma}$ :

$$
0 \longrightarrow \mathbb{Z} \longrightarrow \widetilde{\mathcal{M}} \stackrel{\mu}{\longrightarrow} \mathcal{M} \longrightarrow 1
$$

Note that this extension is natural in the following sense: if $\Sigma^{\prime}$ is a subsurface of $\Sigma$, then we have the following commutative diagram, where $i_{*}$ and $i_{\sharp}$ are the obvious morphisms induced by the inclusion $i$ of $\Sigma^{\prime}$ in $\Sigma$ :

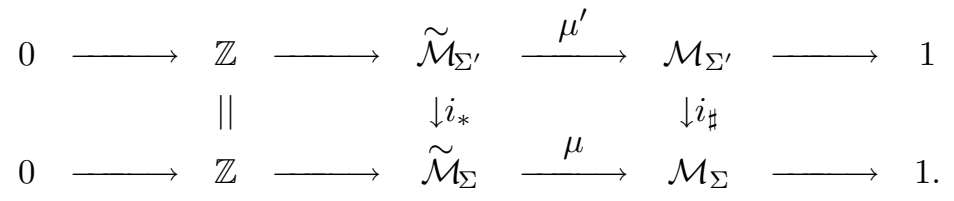

3.2. Presentation of $\tilde{\mathcal{M}}$. Let $\tau_{\alpha}$ be a Dehn twist. We shall construct a canonical lifting $\tilde{\tau}_{\alpha}=\left(\tau_{\alpha}, A\right)$. To do this, consider a neighborhood $V \approx S^{1} \times I_{1} \quad\left(I_{1}=[0,1]\right)$ of $\alpha$ in $\Sigma$ and define $A$ on $(\Sigma \backslash V) \times I$ by $A(\sigma, t)=\varphi(\sigma)$. We have to extend to $V \times I$ the $p_{1}$-structure on $\partial(V \times I)$ which is equal to $\varphi \circ \tau_{\alpha}$ on $V \times 0$ and to $\varphi$ on $(V \times 1 \cup(\partial V) \times I)$.

Now, we paste a copy of $D^{2} \times I_{1}$ to $V$ identifying $S^{1} \times I_{1}$ with $V$.

* $\tau_{\alpha}$ is a diffeomorphism of $S^{1} \times I_{1}$ which can be extended to $D^{2} \times I_{1}$.

* Since $H^{p}\left(D^{2} \times I_{1}, S^{1} \times I_{1} ; \mathbb{Z}\right)=0$ for $p=3$ or 4 , there is one and only one way to extend $\varphi_{\mid V}$ to $D^{2} \times I_{1}$ (up to homotopy).

So, we can define a $p_{1}$-structure $\Phi$ on $D^{2} \times \partial\left(I_{1} \times I\right)$ which extends the given one on $\partial(V \times I)=S^{1} \times \partial\left(I_{1} \times I\right)$ as follow:

$$
\begin{array}{ll}
\Phi(x, \tau, t)=\varphi(x, \tau) & \text { for }(x, \tau, t) \in D^{2} \times \partial I_{1} \times I, \\
\Phi(x, \tau, 0)=\varphi \circ \tau_{\alpha}(x, \tau) & \text { for }(x, \tau) \in D^{2} \times I_{1}, \\
\Phi(x, \tau, 1)=\varphi(x, \tau) & \text { for }(x, \tau) \in D^{2} \times I_{1} .
\end{array}
$$

Since $H^{p}\left(D^{2} \times I_{1} \times I, D^{2} \times \partial\left(I_{1} \times I\right) ; \mathbb{Z}\right)=0$ when $p$ is equal to four or three, there is one and only one way to extend $\Phi$ to $D^{2} \times I_{1} \times I$ up to homotopy. We will denote this extension by $\pi_{\alpha}$, and we define $A$ on $V \times I$ by $A(\sigma, t)=\pi_{\alpha}(\sigma, t)$. This gives a well defined $p_{1}$-structure $A$ on $\Sigma \times I$, and we will denote by $\tilde{\tau}_{\alpha}$ the element $\left(\tau_{\alpha}, A\right)$ of $\tilde{\mathcal{M}}$.

Remark. It is easy to see that when $\alpha$ bounds a disc in $\Sigma$, then $\tilde{\tau}_{\alpha}=1_{\tilde{\mathcal{M}}}$.

Since Dehn twists generate $\mathcal{M}$, the set of all $\tilde{\tau}_{\alpha}$ together with the generator $u$ of $\mathbb{Z}$ generate $\tilde{\mathcal{M}}$. Because $u$ is central in $\widetilde{\mathcal{M}}$, one has the relation $u \tilde{\tau}_{\alpha}=\tilde{\tau}_{\alpha} u$ for any s.c.c. $\alpha$ in $\Sigma$. Theorems 3.7, 3.8 and 3.9 below tell us how braids, lanterns, stars and chains are lifted to $\widetilde{\mathcal{M}}$ by the section $s: \mathcal{M} \rightarrow \widetilde{\mathcal{M}}, s\left(\tau_{\alpha}\right)=\tilde{\tau}_{\alpha}$. Using these results, we obtain from Theorems $\mathrm{A}$ and $\mathrm{B}$ the two following presentations of $\tilde{\mathcal{M}}$. 
Notation. If $m \in \mathcal{M}$ is a word $\tau_{\alpha_{1}} \ldots \tau_{\alpha_{p}}$, we will denote by $\widetilde{m}$ the corresponding word $\tilde{\tau}_{\alpha_{1}} \ldots \tilde{\tau}_{\alpha_{p}} \in \tilde{\mathcal{M}}$.

Theorem 3.5. For any oriented surface $\Sigma, \widetilde{\mathcal{M}}_{\Sigma}$ has the following presentation: generators: $\left\{\tilde{\tau}_{\alpha} / \alpha\right.$ s.c.c. in $\left.\Sigma\right\} \cup\{u\}$ (if $\alpha$ bounds a disc in $\Sigma$, then $\tilde{\tau}_{\alpha}=1$ ). relations: (1) For any s.c.c. $\alpha$ in $\Sigma, \tilde{\tau}_{\alpha} u=u \tilde{\tau}_{\alpha}$.

(2) $\widetilde{T}=1$ for any braids $T_{0}, T_{1}$ and $T_{2_{0}}$.

(3) $\widetilde{C}=u^{12}$ for any chain $C$.

(4) $\widetilde{L}=1$ for any lantern $L$.

If $g \geq 1$, one can replace (3) and (4) by

(5) $\widetilde{E}=u^{12}$ for any star $E$.

With the non-separating curves, we obtain

Theorem 3.6. For any oriented surface $\Sigma$ of genus greater than or equal to two, $\widetilde{\mathcal{M}}_{\Sigma}$ has the following presentation:

generators: $\left\{\tilde{\tau}_{\alpha} / \alpha\right.$ non-separating s.c.c. in $\left.\Sigma\right\} \cup\{u\}$ relations: (1)' For any non-separating s.c.c. $\alpha$ in $\Sigma, \tilde{\tau}_{\alpha} u=u \tilde{\tau}_{\alpha}$.

(2)' $\widetilde{T}=1$ for any braids $T_{0}$ and $T_{1}$ between only non-separating curves.

(3)' $\widetilde{C_{0}}=u^{12}$ for one chain $C_{0}$ with non-separating curves.

(4)' $\widetilde{L_{0}}=1$ for one lantern $L_{0}$ with non-separating curves.

When $g \geq 3$, one can replace (3)' or (4)' by

(5)' $\widetilde{E}_{0}=u^{12}$ for one star $E_{0}$ (with non-separating curves).

3.3. Lifting of the braid relations. The following theorem proves that the braid relations are still true in $\widetilde{\mathcal{M}}_{g, r}$, where $g$ and $r$ are non-negative.

Theorem 3.7. Let $\alpha$ be a s.c.c. in $\Sigma, m=(f, F) \in \widetilde{\mathcal{M}}$ and $\gamma=f(\alpha)$. Then, in $\widetilde{\mathcal{M}}$ we have the relation

$$
\tilde{\tau}_{\gamma}=m \tilde{\tau}_{\alpha} \bar{m} .
$$

Proof. Denote the element $m \tilde{\tau}_{\alpha} \bar{m}$ by $\left(\tau_{\gamma}, \Phi\right)$ (see Figure 28) and consider a neighborhood $V$ of $\gamma$ in $\Sigma$. $V^{\prime}=f^{-1}(V)$ is a neighborhood of $\alpha$.

$$
\begin{array}{|l|l|l|}
\hline F\left(\tau_{\alpha} f^{-1}(\sigma), 3 t\right) & A\left(f^{-1}(\sigma), 3 t-1\right) & F\left(f^{-1}(\sigma), 3(1-t)\right) \\
\hline & \underbrace{}_{\tau_{\gamma} \circ \tau_{\alpha} \circ f^{-1}} & \varphi \circ \tau_{\alpha} \circ f^{-1} \\
\multicolumn{2}{|c|}{\Phi \text { on } \Sigma \times I}
\end{array}
$$

FIGURE 28

First step. We shall homotope $\Phi$ rel $\partial(\Sigma \times I)$ to a $p_{1}$-structure $\Psi$ on $\Sigma \times I$ such that $\Psi(\sigma, t)=\varphi(\sigma)$ for $\sigma \notin V$. To do this, we have to define a $p_{1}$-structure $H$ on $\Sigma \times I \times I$ such that:

$$
\begin{gathered}
H_{\mid \Sigma \times I \times 0}(\sigma, t, 0)=\Phi(\sigma, t), \quad H_{\mid(\Sigma \backslash V) \times I \times 1}(\sigma, t, 1)=\varphi(\sigma), \\
H_{\mid \Sigma \times 0 \times I}(\sigma, 0, \theta)=\varphi \circ \tau_{\gamma}(\sigma), \quad H_{\mid \Sigma \times 1 \times I}(\sigma, 1, \theta)=\varphi(\sigma)=H_{\mid \partial \Sigma \times I \times I}(\sigma, t, \theta) .
\end{gathered}
$$




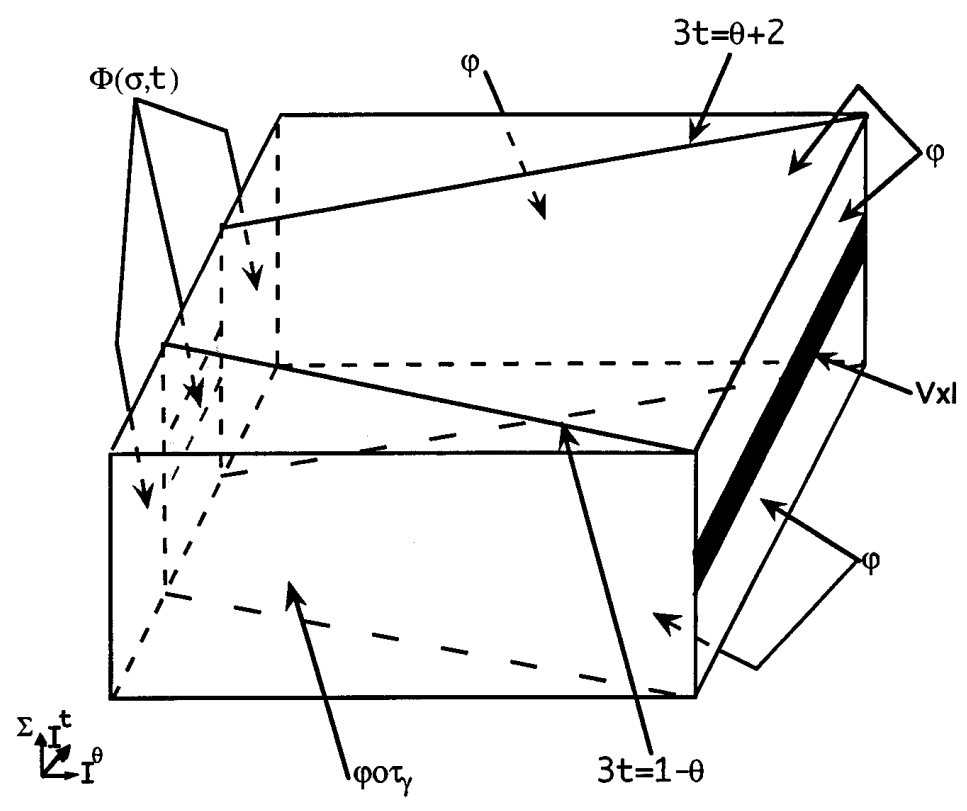

Figure 29

First, we define

$$
H(\sigma, t, \theta)=\mid \begin{array}{ll}
F\left(\tau_{\alpha} f^{-1}(\sigma), 3 t\right) & \text { if } 3 t \leq 1-\theta, \\
F\left(f^{-1}(\sigma), 3(1-t)\right) & \text { if } 3 t \geq 2+\theta, \\
F\left(f^{-1}(\sigma), 1-\theta\right) & \text { if } 1-\theta \leq 3 t \leq 2+\theta \text { and } \sigma \notin V .
\end{array}
$$

Then, it remains us to extend $H$ to $W=\{(\sigma, t, \theta) \in V \times I \times I$ such that $1-\theta \leq$ $3 t \leq 2+\theta\}$, which is diffeomorphic to $V \times I \times I$ (see Figure 29; we will identify $W$ with $V \times I \times I) . \quad H$ is given on $M=(\partial(V \times I) \times I) \cup(V \times I \times 0)$. Since $H^{4}(W, M)=H^{3}(W, M)=0$, there is one and only one way to extend $H$ to $W$ up to homotopy. We define $\Psi$ by $\Psi(\sigma, t)=H(\sigma, t, 1)$.

Second step. To show that $\left(\tau_{\gamma}, \Psi\right)=\tilde{\tau}_{\gamma}$, we only need to look at the $p_{1}$-structure on $V \times I$. So, from now on, $\varphi$ is considered as a $p_{1}$-structure on $V$ and $V^{\prime}$, and $f$ is considered as a diffeomorphism from $V^{\prime}$ to $V$. Consider two copies of $D^{2} \times I_{1}$ (one is denoted $D^{\prime 2} \times I_{1}$ ) and paste them on $V$ and $V^{\prime}$. Extend $f$ to a diffeomorphism from $D^{\prime 2} \times I_{1}$ to $D^{2} \times I_{1}$, and $\varphi$ to a $p_{1}$-structure on $D^{2} \times I_{1}$ and $D^{\prime 2} \times I_{1}$. Then, we define a $p_{1}$-structure $\Xi$ on $\partial\left(D^{2} \times I_{1} \times I\right)$ by

$$
\Xi(x, \tau, t)= \begin{cases}\Psi(x, \tau, t) & \text { if } x \in S^{1}, \\ \varphi \circ \tau_{\gamma}(x, \tau) & \text { if } t=0, \\ \varphi(x, \tau) & \text { if } t=1 \text { or } \tau \in\{0,1\} .\end{cases}
$$

To prove that $\left(\tau_{\gamma}, \Psi\right)=\tilde{\tau}_{\gamma}$, it is enough to show that $\Xi$ can be extended to $D^{2} \times I_{1} \times I$ (see definition of $\tilde{\tau}_{\gamma}$ in 3.2). To do this, we shall find a $p_{1}$-structure $K$ on $D^{2} \times I_{1} \times I \times I$ such that $K_{\mid \partial\left(D^{2} \times I \times I\right) \times 1}$ coincides with $\Xi$. Then, $K_{\mid D^{2} \times I \times I \times 1}$ will be the extension of $\Xi$.

We recall that $F$ is a $p_{1}$-structure on $\Sigma \times I$. We shall extend it to $D^{\prime 2} \times I_{1} \times I$. We consider its restriction to $V^{\prime} \times I=S^{1} \times I_{1} \times I$, which we will also denote $F$. 
Since $H^{4}\left(D^{2} \times I_{1} \times I,\left(S^{1} \times I_{1} \times I\right) \cup\left(D^{2} \times I_{1} \times \partial I\right)\right)=0$, there exists a $p_{1}$-structure $\hat{F}$ on $D^{\prime 2} \times I_{1} \times I$ such that $\hat{F}_{\mid S^{1} \times I_{1} \times I}=F, \hat{F}_{\mid D^{\prime 2} \times I_{1} \times 0}=\varphi \circ f$ and $\hat{F}_{\mid D^{\prime 2} \times I_{1} \times 1}=\varphi$.

Now, we can define $K$ on $M^{\prime}=\overline{\partial\left(D^{2} \times I_{1} \times I \times I\right) \backslash D^{2} \times I \times I \times 1}$ by

$K(x, \tau, t, \theta)= \begin{cases}\pi_{\alpha}\left(f^{-1}(x, \tau), t\right) & \text { if } \theta=0, \\ H(x, \tau, t, \theta) & \text { if } x \in S^{1} \quad \text { if } t=1 \text { or } \tau \in\{0,1\}, \\ \hat{F}\left(f^{-1}(x, \tau), 1-\theta\right) & \left.\text { if } t=S^{1} \times I_{1} \times I \times I\right), \\ \hat{F}\left(\tau_{\alpha} f^{-1}(x, \tau), 1-\theta\right) & \text { if } t=0 .\end{cases}$

One can check that $K$ coincides with $\Xi$ on $\partial\left(D^{2} \times I_{1} \times I\right) \times 1$; since when $p=3$ or 4 we have $H^{p}\left(D^{2} \times I_{1} \times I \times I, M^{\prime}\right)=0 \mathrm{~K}$ can be extended in a unique way up to homotopy to $D^{2} \times I_{1} \times I \times I$.

\subsection{Lifting of the lantern relations.}

Theorem 3.8. Let $\Sigma$ be a sphere with four holes. If $\alpha_{1}, \ldots, \alpha_{4}, \beta_{1}, \beta_{2}$ and $\beta_{3}$ are the curves shown in Figure 1 , then one has in $\tilde{\mathcal{M}}_{\Sigma}$ :

$$
\tilde{\tau}_{\alpha_{1}} \tilde{\tau}_{\alpha_{2}} \tilde{\tau}_{\alpha_{3}} \tilde{\tau}_{\alpha_{4}}=\tilde{\tau}_{\beta_{3}} \tilde{\tau}_{\beta_{2}} \tilde{\tau}_{\beta_{1}} \text {. }
$$

Consequence. Since the extension $(* *)$ is natural, the lantern relations will be true in $\widetilde{\mathcal{M}}_{\Sigma}$ for any surface $\Sigma$.

Proof. Since $\tau_{\alpha_{1}} \tau_{\alpha_{2}} \tau_{\alpha_{3}} \tau_{\alpha_{4}}=\tau_{\beta_{3}} \tau_{\beta_{2}} \tau_{\beta_{1}}$, there exists an integer $n$ such that

$$
\tilde{\tau}_{\alpha_{1}} \tilde{\tau}_{\alpha_{2}} \tilde{\tau}_{\alpha_{3}} \tilde{\tau}_{\alpha_{4}}=\tilde{\tau}_{\beta_{3}} \tilde{\tau}_{\beta_{2}} \tilde{\tau}_{\beta_{1}} u^{n} \text {. }
$$

Consider an embedding of $\Sigma$ in a surface $\Sigma^{\prime}$ such that $\alpha_{4}$ bounds a disc in $\Sigma^{\prime}$. Then, we have $\tilde{\tau}_{\alpha_{4}}=1$ and $\beta_{k}$ is isotopic to $\alpha_{k}$, thus $\tilde{\tau}_{\beta_{k}}=\tilde{\tau}_{\alpha_{k}}$ and $\tilde{\tau}_{\alpha_{1}} \tilde{\tau}_{\alpha_{2}} \tilde{\tau}_{\alpha_{3}}=$ $\tilde{\tau}_{\alpha_{3}} \tilde{\tau}_{\alpha_{2}} \tilde{\tau}_{\alpha_{1}} u^{n}$, which implies that $n=0$.

\subsection{Lifting of the star and chain relations.}

Theorem 3.9. Let $\Sigma$ be a surface of genus one with three holes. If $\alpha_{1}, \alpha_{2}, \alpha_{3}, \beta$, $\delta_{1}, \delta_{2}$ and $\delta_{3}$ are the curves shown in Figure 2, then, in $\widetilde{\mathcal{M}}_{\Sigma}$, we have the relation:

$$
\left(\tilde{\tau}_{\alpha_{1}} \tilde{\tau}_{\alpha_{2}} \tilde{\tau}_{\alpha_{3}} \tilde{\tau}_{\beta}\right)^{3}=\tilde{\tau}_{\delta_{1}} \tilde{\tau}_{\delta_{2}} \tilde{\tau}_{\delta_{3}} u^{12} .
$$

Consequence. For any surface $\Sigma$ and any stars $E$ or chain $C$ in $\Sigma$, in $\widetilde{\mathcal{M}}_{\Sigma}$ we have the relation $\widetilde{E}=\widetilde{C}=u^{12}$.

Proof. Since the star relations are verified in $\mathcal{M}$, there exists an integer $n$ such that $\left(\tilde{\tau}_{\alpha_{1}} \tilde{\tau}_{\alpha_{2}} \tilde{\tau}_{\alpha_{3}} \tilde{\tau}_{\beta}\right)^{3}=\tilde{\tau}_{\delta_{1}} \tilde{\tau}_{\delta_{2}} \tilde{\tau}_{\delta_{3}} u^{n}$. Consider an embedding of $\Sigma$ in $S^{1} \times S^{1}$ and denote by $\alpha$ the common image of $\alpha_{1}, \alpha_{2}$ and $\alpha_{3}$. Since each of the three curves $\delta_{1}, \delta_{2}$ and $\delta_{3}$ bounds a disc in $S^{1} \times S^{1}$, the relation becomes

$$
\left(\tilde{\tau}_{\alpha} \tilde{\tau}_{\beta} \tilde{\tau}_{\alpha}\right)^{4}=u^{n}
$$

In order to prove that $n=12$, we shall use the action of $\widetilde{\mathcal{M}}_{S^{1} \times S^{1}}$ on $V_{p}=$ $V_{p}\left(S^{1} \times S^{1}\right)$ induced by the TQFT developped in [BHMV2].

First, let us describe $V_{p}$. We suppose that $p \geq 4$ and we consider the ring $k_{p}$ define by

$$
k_{p}=\mathbb{Z}\left[\frac{1}{p}, A, \kappa\right] /\left(\varphi_{2 p}(A), \kappa^{6}-u\right)
$$


where $\varphi_{2 p}(A)$ is the $2 p$-th cyclotomic polynomial and $u=A^{-6-\frac{p(p+1)}{2}}$. Let us denote by $K\left(D^{2} \times S^{1}\right)$ (resp. $\left.K\left(S^{1} \times D^{2}\right)\right)$ the Jones-Kauffman module of $D^{2} \times S^{1}$ (resp. $\left.S^{1} \times D^{2}\right)$ over $k_{p}$. There is a bilinear form

$$
\langle,\rangle: K\left(D^{2} \times S^{1}\right) \times K\left(-S^{1} \times D^{2}\right) \longrightarrow k_{p}
$$

which associates to a pair of banded links $L, L^{\prime}$ in $D^{2} \times S^{1}$ and $S^{1} \times D^{2}$ the Kauffman bracket of $L \cup L^{\prime}$ in $S^{3}$, using the standard decomposition

$$
D^{2} \times S^{1} \underset{S^{1} \times S^{1}}{\cup} S^{1} \times\left(-D^{2}\right)
$$

of $S^{3}$. Then, $V_{p}$ is the quotient of $K\left(D^{2} \times S^{1}\right)$ by the left kernel. It is shown in [BHMV1] that $V_{p}$ is a $k_{p}$-module of dimension [(p-1)/2].

The Dehn twist $\tau_{\alpha}$ induces an obvious endomorphism $t$ on $K\left(D^{2} \times S^{1}\right)$ which descends to $V_{p}$. Similarly, $\tau_{\beta}$ induces an endomorphism on $K\left(-S^{1} \times D^{2}\right)$. Considering its adjoint, we obtain a new endomorphism $t^{\prime}$ on $V_{p}$. Then, the actions of $\tilde{\tau}_{\alpha}$ and $\tilde{\tau}_{\beta}$ on $V_{p}$ coincide respectively with $t$ and $t^{\prime}$. By using methods and results of [BHMV1], one can check that $\left(t t^{\prime} t\right)^{2}=-A^{-6} A^{-p(p-1) / 2} \operatorname{Id}_{V_{p}}$ when $p$ is odd. So, since $u$ acts as the multiplication by the scalar $\kappa,(\star)$ implies $A^{-12} A^{-p(p-1)}=\kappa^{n}$ in $k_{p}$. Suppose now that $p \equiv-1 \bmod 4$. Then, $\kappa^{n}=\kappa^{12}$ and, since $\kappa^{6}=A^{-6}$, we obtain $1=\kappa^{6(n-12)}=A^{-6(n-12)}$. Moreover, since $A$ is a primitive $2 p$-th root of unity, one has $-6(n-12) \equiv 0(2 p)$ for all integers $p \geq 4$ such that $p \equiv-1 \bmod 4$. This proves the required equality $n=12$.

\section{On central extensions of $\mathcal{M}_{\Sigma}$}

Let $\Sigma$ be a surface of genus $g \geq 0$ with $r$ boundary components $(r \geq 0)$. Recall that $S_{\Sigma}$ is a group generated by $\left\{a_{\alpha} / \alpha\right.$ a non-separating s.c.c. in $\left.\Sigma\right\}$ and defined by the relations $T_{0}$ and $T_{1}$. Let $\mathcal{T}_{\Sigma}$ be the subgroup of $\widetilde{\mathcal{M}}_{\Sigma}$ generated by $\left\{\tilde{\tau}_{\alpha} / \alpha\right.$ s.c.c. in $\Sigma\} \cup\left\{u^{12}\right\}$. In this section, we shall prove the following theorem:

Theorem 4.1. (i) Considering the restriction of $\mu$ to $\mathcal{T}_{\Sigma}$, we have the natural central extension

$$
0 \longrightarrow \mathbb{Z} \longrightarrow \mathcal{T}_{\Sigma} \stackrel{\mu}{\longrightarrow} \mathcal{M}_{\Sigma} \longrightarrow 1
$$

(ii) If $g \geq 2, \mathcal{T}_{\Sigma}$ is generated by $\left\{\tilde{\tau}_{\alpha} / \alpha\right.$ non-separating s.c.c. in $\left.\Sigma\right\}$.

(iii) One can define natural homorphisms $\varphi: S_{\Sigma} \rightarrow \mathcal{T}_{\Sigma}$ and $\psi: S_{\Sigma} \rightarrow \mathbb{Z}$ by $\varphi\left(a_{\alpha}\right)=\tilde{\tau}_{\alpha}$ and $\psi\left(a_{\alpha}\right)=1$.

When $g \geq 3, \varphi$ and $\psi$ induce an isomorphism between $S_{\Sigma}$ and $\mathcal{T}_{\Sigma} \times \mathbb{Z}$.

When $g=2, \varphi$ is an isomorphism from $S_{\Sigma}$ to $\mathcal{T}_{\Sigma}$.

(iv) When $g \geq 3$, the extension $\left(^{*}\right)$ is the universal central extension of $\mathcal{M}_{\Sigma}$.

Clearly, this theorem proves Theorem C (the functorial properties are obvious) and implies the following two corollaries:

Corollary 4.2. The kernel of the quotient map $S_{\Sigma} \rightarrow \mathcal{M}_{\Sigma}$ is isomorphic to $\mathbb{Z}$ (generated by $C_{0}$ ) when $g=2$ and to $\mathbb{Z} \oplus \mathbb{Z}$ (generated by $L_{0}$ and $C_{0}$ ) when $g \geq 3$.

Corollary 4.3. Suppose that $g \geq 3$. Then, $\mathcal{T}_{\Sigma}$ admits a presentation with generators $\left\{a_{\alpha} / \alpha\right.$ non-separating s.c.c. in $\left.\Sigma\right\}$ and the relations (I)' and (II)' (braids $T_{0}$ and $T_{1}$ and a lantern $L$ with only non-separating curves). 
Proof of Theorem 4.1. (i) is clear since the kernel of $\mu_{\mid \mathcal{T}_{\Sigma}}$ is generated by $u^{12}$.

To prove (ii), first one can see, by considering a chain $C$ without separating curves and using Theorem 3.9, that $u^{12}$ is in the subgroup of $\widetilde{\mathcal{M}}_{\Sigma}$ generated by $\left\{\tilde{\tau}_{\alpha} / \alpha\right.$ a non-separating s.c.c. in $\left.\Sigma\right\}$. Then, we can conclude exactly as in the proof of Lemma 2.5.

Since braid relations are verified in $\widetilde{\mathcal{M}}_{\Sigma}$, the map $\varphi$ is a morphism. Furthermore, (ii) implies that $\varphi$ is onto when $g \geq 2$. Now, we shall determine $\operatorname{Ker} \varphi$. Denote by $q: S_{\Sigma} \rightarrow \mathcal{M}_{\Sigma}$ the quotient map given by Theorem B. First, we suppose that $g \geq 3$. Then, $\operatorname{Ker} q$ is generated by two elements $C_{0}$ and $L_{0}$ coming from chains and lanterns. So, considering the commutative diagram

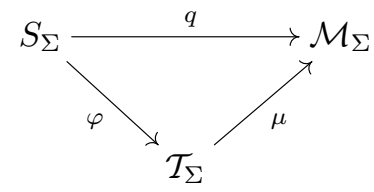

we see that $\operatorname{Ker} \varphi \subset\left\langle C_{0}, L_{0}\right\rangle$. Since this group is abelian (Theorem 1.6) and $\varphi\left(L_{0}\right)=1$ and $\varphi\left(C_{0}\right)=u^{12}$, we have $\operatorname{Ker} \varphi=\left\langle L_{0}\right\rangle$. But since $\psi$ is the abelianized morphism and $\psi\left(L_{0}\right)=1$, we have $\left\langle L_{0}\right\rangle=\mathbb{Z}$ and we obtain the following exact sequence:

$$
0 \longrightarrow \mathbb{Z} \longrightarrow S_{\Sigma} \stackrel{\varphi}{\longrightarrow} \mathcal{T}_{\Sigma} \longrightarrow 1
$$

Furthermore, since $\psi: S_{\Sigma} \rightarrow \mathbb{Z}$ is a section, this sequence splits. When $g=2$, Ker $q$ is cyclic generated by $C_{0}$. Thus, $\varphi$ is an isomorphism.

(iv) was shown by Harer in $[\mathrm{H}]$.

\section{The GENUS ONE CASE}

The goal of this section is to prove Lemma 2.6 and the fact that there is no epimorphism from $S_{1, r}$ to $\mathcal{M}_{1, r}$ if $r \geq 2$. In fact, we shall prove stronger results.

Lemma 5.1. Considering the curves shown on Figure $30, \mathcal{M}_{1, r}$ is generated by the twists $\tau_{\alpha_{i}}, \tau_{\beta}$ and $\tau_{\delta_{j}}$ for $j \geq 2$.

Proof. We shall use an inductive argument. Capping off $\delta_{r}$ by a disc with center $p$, one can obtain the following two exact sequences (see $[\mathrm{B}]$ and $[\mathrm{H}]$ ):

$$
\begin{gathered}
0 \longrightarrow \mathbb{Z} \stackrel{f_{1}}{\longrightarrow} \mathcal{M}_{1, r} \stackrel{f_{2}}{\longrightarrow} \mathcal{N}_{1, r-1} \longrightarrow 1, \\
0 \longrightarrow \pi_{1}\left(\Sigma_{1, r-1}, p\right) \stackrel{f_{3}}{\longrightarrow} \mathcal{N}_{1, r-1} \stackrel{f_{4}}{\longrightarrow} \mathcal{M}_{1, r-1} \longrightarrow 1,
\end{gathered}
$$

where $\mathcal{N}_{1, r-1}$ is the group of isotopy classes of orientation preserving diffeomorphisms of $\Sigma_{1, r-1}$ which are the identity on the boundary and which leave fixed the base point $p$. The four morphisms $f_{k}$ are defined as follows:

$\rightarrow f_{1}(1)=\delta_{r}$.

$\rightarrow f_{2}$ is obtained by extending each map over the disc by the identity.

$\rightarrow$ If $\alpha$ is a loop based on $p$ and $\alpha^{\prime}$ and $\alpha^{\prime \prime}$ the two boundary components of a neighborhood of $\alpha$, then $f_{3}(\alpha)=\tau_{\alpha^{\prime}} \tau_{\alpha^{\prime \prime}}^{-1}$ (here and below, we will denote by the same letter a curve and its homotopy class).

$\rightarrow f_{4}$ is defined by forgetting the base point $p$. 


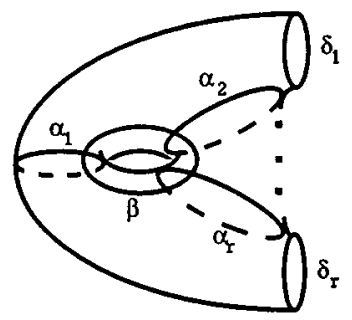

FigURE 30

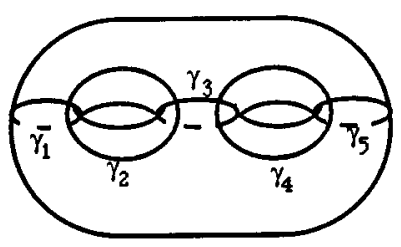

FiguRE 31

It is known that $\mathcal{M}_{1,1}$ is generated by $\tau_{\alpha_{1}}$ and $\tau_{\beta}$. Suppose inductively that $\mathcal{M}_{1, r-1}$ is generated by $\tau_{\alpha_{1}}, \ldots, \tau_{\alpha_{r-1}}, \tau_{\beta}, \tau_{\delta_{2}}, \ldots, \tau_{\delta_{r-1}}$. Then, $\mathcal{N}_{1, r-1}$ is generated by these twists and $\operatorname{Im} f_{3}$. Furthermore, $\pi_{1}\left(\Sigma_{1, r-1}, p\right)$ is generated by the homotopy classes of the curves $\alpha_{1}, \ldots, \alpha_{r-1}$ and $\beta$. If we denote by $\alpha_{i}^{\prime}$ the curve $\tau_{\beta} \tau_{\alpha_{1}} \tau_{\alpha_{r}}^{-1} \tau_{\beta}^{-1}\left(\alpha_{i}\right)$, then one can check that $f_{3}$ maps $\alpha_{i}$ into $\tau_{\alpha_{i}} \tau_{\alpha_{i}^{\prime}}^{-1}$, and if $\beta^{\prime}=\tau_{\alpha_{1}} \tau_{\beta}\left(\alpha_{r}\right)$, then $f_{3}(\beta)=\tau_{\beta} \tau_{\beta^{\prime}}^{-1}$. Thus, since $\tau_{\alpha_{i}^{\prime}}=\tau_{\beta} \tau_{\alpha_{1}} \tau_{\alpha_{r}}^{-1} \tau_{\beta}^{-1}\left(\tau_{\alpha_{i}}\right)$ and $\tau_{\beta^{\prime}}=\tau_{\alpha_{1}} \tau_{\beta}\left(\tau_{\alpha_{r}}\right)$, the group $\mathcal{N}_{1, r-1}$ is generated by $\tau_{\alpha_{1}}, \ldots, \tau_{\alpha_{r-1}}, \tau_{\beta}, \tau_{\delta_{2}}, \ldots, \tau_{\delta_{r-1}}$ and $\tau_{\alpha_{r}}$.

Finally, we obtain the required result for $\mathcal{M}_{1, r}$ using the first exact sequence.

Proposition 5.2. Suppose that $r \geq 2$. Then, the twists along non-separating curves and $r-2$ boundary curves do not generate $\mathcal{M}_{1, r}$.

Proof. Referring to Figure 30, suppose that $\tau_{\delta_{1}}$ and $\tau_{\delta_{2}}$ can be decomposed into a product of twists along non-separating curves or the curves $\delta_{3}, \ldots, \delta_{r}$. First, let $\alpha$ be a non-separating curve in $\Sigma=\Sigma_{1, r}$. There is a diffeomorphism $h$ of $\Sigma$ which sends $\alpha_{1}$ on $\alpha$. Lemma 5.1 tells us that $h$ can be decomposed into a product of twists along the curves $\alpha_{1}, \ldots, \alpha_{r}, \beta, \delta_{2}, \ldots, \delta_{r}$. One has $\tau_{\alpha}=h \tau_{\alpha_{1}} \bar{h}$; since Dehn twists along boundary curves are central in $\mathcal{M}_{1, r}$, one still has $\tau_{\alpha}=h^{\prime} \tau_{\alpha_{1}} \overline{h^{\prime}}$, where $h^{\prime}$ is obtain from $h$ by removing the boundary twits in its decomposition. Thus, one can suppose that $\tau_{\delta_{1}}$ and $\tau_{\delta_{2}}$ are decomposed into a product of twists along the curves $\alpha_{1}, \ldots, \alpha_{r}, \beta, \delta_{3}, \ldots, \delta_{r}$.

Now, let $\varphi: \mathcal{M}_{1, r} \rightarrow \mathcal{M}_{2,0}$ be the morphism obtained by capping off each of the boundary components $\delta_{j}$ for $j \geq 3$ with a disc and gluing a cylinder onto $\delta_{1}$ and $\delta_{2}$. Then, if $\gamma_{1}, \ldots, \gamma_{5}$ are the curves shown in Figure 31, one has $\varphi\left(\tau_{\beta}\right)=$ $\tau_{\gamma_{2}}, \varphi\left(\tau_{\alpha_{i}}\right)=\tau_{\gamma_{1}}$ for $i \neq 2, \varphi\left(\tau_{\alpha_{2}}\right)=\tau_{\gamma_{3}}, \varphi\left(\tau_{\delta_{1}}\right)=\varphi\left(\tau_{\delta_{2}}\right)=\tau_{\gamma_{5}}$ and $\varphi\left(\tau_{\delta_{j}}\right)=1$ for $j \geq 3$. Thus, our hypothesis implies that $\tau_{\gamma_{5}}$ can be decomposed into a product of twists along the three curves $\gamma_{1}, \gamma_{2}$ and $\gamma_{3}$. Then, since $\tau_{\gamma_{1}}, \ldots, \tau_{\gamma_{5}}$ generate 
$\mathcal{M}_{2,0}$, one only needs $\tau_{\gamma_{1}}, \ldots, \tau_{\gamma_{4}}$ to generate $\mathcal{M}_{2,0}$. This is false according to Humphries $([\mathrm{Hu}])$.

\section{ACKNOWLEDGEMENTS}

This article is a short version of my thesis $([\mathrm{G}])$ written under the supervision of Professor P. Vogel. I am grateful to him for his help and advices. I want to thank Professors C. Blanchet and N. Habegger as well, for many useful discussions.

\section{REFERENCES}

[A1] M. Atiyah. Topological quantum field theories, Publ. Math. IHES 68, 175-186 (1989). MR 90e:57059

[A2] M. Atiyah. On framings of 3-manifolds, Topology 29, 1-7 (1990). MR 91g:57025

[B] J. Birman. Braids, Links, and Mapping Class Groups, Annals of Math. Studies 82, Princeton Univ. Press (1975). MR 51:11477; MR 54:13894

[BHMV1] C. Blanchet, N. Habegger, G. Masbaum, P. Vogel. Three-manifold invariants derived from the Kauffman bracket, Topology 31, 685-699 (1992). MR 94a:57010

[BHMV2] C. Blanchet, N. Habegger, G. Masbaum, P. Vogel. Topological quantum field theories derived from the Kauffman bracket, Topology 34, 883-927 (1995). CMP 96:04

[D] M. Dehn. Die Gruppe der Abbildungsklassen, Acta Math. 69, 135-206 (1938).

[G] S. Gervais. Etude de certaines extensions centrales du "mapping class group" des surfaces orientables, thèse, Université de Nantes, janv. 1994.

$[\mathrm{H}] \quad$ J. Harer. The second homology group of the mapping class group of an orientable surface, Invent. Math. 72, 221-239 (1983). MR 84g:57006

[Hu] S. Humphries. Generators for the mapping class group, Lecture Notes in Math. 722, Springer, Berlin, 44-47 (1979). MR 80i:57010

[H-T] A. Hatcher, W. Thurston. A presentation for the mapping class group of a closed orientable surface, Topology 19, 221-237 (1980). MR 81k:57008

[J] D. Johnson. Homeomorphisms of a surface which act trivially on homology, Proc. of Amer. Math. Soc. 75, 119-125 (1979). MR 80h:57008

[L1] W.B.R. Lickorish. A finite set of generators for the homeotopy group of a 2-manifold, Proc. Cambridge Philos. Soc. 60, 769-778 (1964). MR 30:1500

[L2] W.B.R. Lickorish. On the homeotopy group of a 2-manifold (Corrigendum), Proc. Cambridge Philos. Soc. 62, 679-681 (1966). MR 34:799

[M-R] G. Masbaum, J. Roberts. On central extensions of mapping class groups, Math. Annalen. 302, 131-150 (1995). CMP 95:12

[S] R. Stong. Notes on cobordism theory, Princeton (1968). MR 40:2108

[Sw] R.M. Switzer. Algebraic topology-homotopy and homology, Berlin, Heidelberg, New York, Springer (1975). MR 52:6695

[W] B. Wajnryb. A simple presentation for the mapping class group of an orientable surface, Israel Journal of Math. 45, 157-174 (1983). MR 85g:57007

URA N ${ }^{\circ} 758$ du C.N.R.S., Université de Nantes, Département de Mathématiques, 2 Rue de la Houssinière, F-44072 Nantes Cedex 03, France

E-mail address: gervais@math.univ-nantes.fr 